\title{
THE NURSES GROUP POSTER SESSION
}

NP001

Family members' experiences of different caring

organizations during allogeneic hematopoietic stem cells transplantation - A qualitative interview study

K. Bergkvist ${ }^{1, *}$, J. Larsen ${ }^{2}$, U.-B. Johansson ${ }^{1}$, J. Mattsson ${ }^{3}$, B. Fossum ${ }^{1}$

${ }^{1}$ Sophiahemmet University, ${ }^{2}$ Red Cross University College, ${ }^{3}$ Oncology and Pathology, Karolinska Institutet, Stockholm, Sweden

Introduction: Home care after allogeneic hematopoietic stem cell transplantation (HSCT) has been an option for over 15 years. Earlier studies have shown that home care is safe and has medical advantages. Because of the complex and intensive nature of the HSCT, most patients require a family member to assist them with their daily living. Today, there is a limited knowledge about family members' experiences in different care settings. Therefore, the aim of this study was to describe family members' life situation and experiences of care in two different care settings, the patient's home or in hospital during the acute post-transplantation phase after (HSCT).

Method: Data were collected through semi-structured interviews with 14 family members (seven women and seven men). An inductive qualitative content analysis was used to analyze the data.

Results: The majority of the family members $n=10$ had experiences from home care. The main category Being me being us in an uncertain time was identified, with following five generic categories: To receive the information I need, To meet a caring organization, To be in different care settings, To be a family member and To have a caring relationship. The findings show that family members of a patient undergoing a HSCT live in uncertainty. Positive experiences such as freedom and security from home care were identified. The competence and support from the healthcare professionals was profound. Different strategies (adjusting, having hope and live in the present) used to balance the uncertainty.

Conclusion: The healthcare professionals need to identify psychosocial problems, and integrate the psychosocial support for the family to prevent uncertainty during HSCT, regardless of the care setting.

Disclosure of Interest: None declared.

\section{NP002}

Meeting the needs of family carers in the bone marrow transplant setting

\section{K. Wilczek ${ }^{1, *}$, H. Mangan ${ }^{1}$, C. Barnett ${ }^{2}$, K. White ${ }^{2}$}

${ }^{1}$ Institute of Haematology, Royal Prince Alfred Hospital, ${ }^{2}$ Cancer Nursing Research Unit, University of Sydney, Camperdown, Australia

Introduction: Improvements in treatment and supportive care have led to shorter duration in hospitalization for individuals receiving stem cell transplant. Discharge is dependent on access to ongoing support and care from family members. Family members are being required to play a more active role in supporting the patients clinical care in the home environment, provide oversight of the patients medical progress and manage complex medication regimens. Few family members will have had experience in providing this care previously. Caregiver fatigue due to the many competing physical, social and emotional demands of caring for BMT patients is a risk.
This abstract outlines the development and testing of an education program for family carers of individuals about to undergo BMT. The project aimed to increase carer confidence in supporting newly discharged blood and marrow transplant (BMT) recipients through an interactive education program. Method: Evaluation methodology was used to examine the impact on carer confidence. Brief questionnaires to assess level of confidence were implemented pre- and post- each session; questions were specific to the content of that session. Following completion of the program an overall evaluation survey was also completed. The education sessions were developed drawing on evidence from literature, unit specific practice guidelines and the team's expertise. Carers of individuals who were about to receive, or currently receiving BMT, were invited to attend the education program. Completing the evaluation was not a program requirement.

Results: Up to 14 carers attended each session. Data was grouped and analysed using SPSS. Wilcoxon (2 related pairs) tests explored changes in confidence for each session. Effect sizes ranged from -0.75 to -0.90 indicating large to very large improvements in confidence from pre- to post- education sessions. Overall satisfaction with presentation and content of the sessions was also very high $(M=4.57, S D=.33$, range 3.9-5). Conclusion: Carers were noticeably more confident about their knowledge base around BMT, and how they should care for themselves, after attending the education program. Qualitative feedback was overwhelmingly positive. Constructive feedback on content will guide further refinement. The team is exploring other mediums to deliver this program.

Disclosure of Interest: None declared.

\section{NP003}

The role of transplant nursing in the taking charge of the care and nutritional context in neutropenic patients

L. Pasquali ${ }^{1, *}$, L. Cappucciati ${ }^{2}$, A. Contini ${ }^{3}$, C. Merlini ${ }^{3}$, V. M. Quitadamo ${ }^{2}$, M. A. Zucconi ${ }^{2}$, G. Pattini ${ }^{2}$, S. Fava ${ }^{\prime}$, S. Gandolfi ${ }^{4}$, L. Cavanna ${ }^{5}, D$. Vallisa ${ }^{6}$

${ }^{1}$ NURSE, Piacenza, Italy, ${ }^{2}$ Hematology, ${ }^{3}$ hospital, NURSE, ${ }^{4}$ hospital, Statistician, ${ }^{5}$ Oncology, ${ }^{6}$ Hematology, Head physician, Piacenza, Italy

Introduction: Nutrition is part of the main competences of nurses involved in hematological neutropenic patients' care. We have considered this issue within the Hematology and Bone Marrow Transplant ward (which has 14 beds) at Ospedale Civile in Piacenza, with three main purposes: evaluating the nurses' knowledge on neutropenic patients' nutritional assessment, evaluating patients' satisfaction, and detecting possible connections between the patients' psycho-physical conditions and their level of nutritional interest while they are hospitalized.

Method: From 20.08.2015 to 30.09.2015 ten nurses were asked to complete a multiple choice questionnaire. Over the same period thirty-one patients were administered the Distress Thermometer and they were given a questionnaire on nutritional issues (both questionnaires have been created by us).

Results: The Distress Thermometer shows that physical problems (Tab. 2) deeply affect nutrition in neutropenic patients. Combining the patients' answers to the Distress Thermometer to the questionnaire results, we have found out that there is a considerable connection: concern and 
Tab. 1: Emotional Problems

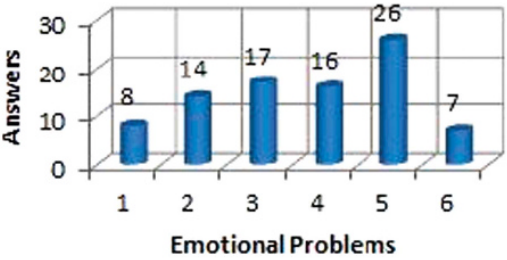

Legend:1.Depression; 2.Fears; 3. Irritability; $4.5 a$ dness; 5 . Concern; 6 .Loss of interest in usual activities

Tab. 2: Physical Problems

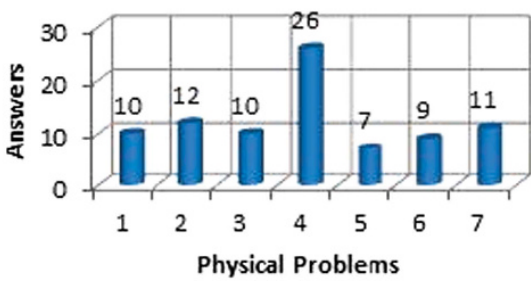

Chart 1: In your opinion is better to eat hospital food or bring food from home?

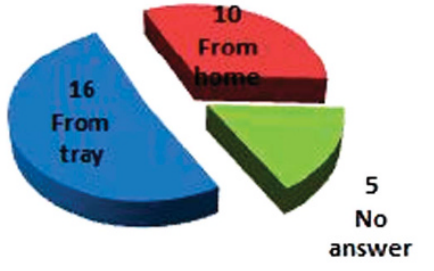

Legend:1.Sleep problems, 2.Pain,3.Nausea, 4

Fatigue, 5.Mouth ulcers, 6.Food problems, 7. Constipation

depression influence the food choice. Furthermore, when the patients are depressed, they often ask their family to bring them some food from home, so as to maintain some kind of family routine (Tab. 1-Chart 1). This research work also confirms the existing connection between pain, nausea, mouth ulcers and food choice. As far as nurses are concerned, they appear to be ready to provide neutropenic patients with nutrition education. However, they would like to work with the support of a nutritionist and they feel they need to get more training on the issue of patients' nutrition.

Conclusion: This study has allowed us to undertake two important actions: the first one is to use the Distress Thermometer in order to strengthen the psychologist's involvement in patients' nutritional context. The second one is to start a training programme aiming at nutrition education, possibly including the nutritionist's support. Considering that the sampling is not sufficient for statistical purposes, the future goal is to propose it as a European multicenter study.

Disclosure of Interest: None declared.

NP004

JOB SATISFACTION AMONG NURSES WORKING IN ONCOLOGY UNITS IN A TERTIARY SPECIALIZED HOSPITAL: A DESCRIPTIVE CROSS SECTIONAL APPROACH M. Alali ${ }^{1, *}$

${ }^{7}$ Oncology Center, KFS\&RC, Riyadh, Saudi Arabia

Introduction: Oncology nurses face different daily challenges to provide high standard of quality care for patients with cancer; those challenges may include shortage of staff, patients' acuity, workload, and work environment, In Saudi Arabia, scarce of studies discussed oncology nurses' job satisfaction in specific, whereas some studies discussed nurses' job satisfaction generally without referring to a specialty area. Nurses' perceived level of job satisfaction at oncology units need to be well understood, to formulate a clear description of the actual satisfaction level and consequently identify areas of improvements.

Method: A descriptive, cross-sectional design was used, with data collected from oncology nurses using survey $(N=202)$, collected. Chi square was used to explore the relationship of level of satisfaction in term of coping with stress with nurses' gender, specialty, and unit role. In addition, Pearson correlation was used to explore the relationship between level of satisfaction in term of coping with stress and number of years of experience.

Results: Nurses' perceived their professional work environment as somewhat satisfying (Mean=2.57, SD =0.47). Additionally, nurses' gender, specialty, and nurses' role in the unit were significantly correlated with the level of satisfaction in term of coping with stress $X 2 \quad(1, N=202)=120.48$, $P<0.001), \quad X 2 \quad(5, \quad \mathrm{~N}=202)=309.34, \quad P<0.001), \quad \mathrm{X} 2$ (4, $\quad N=202)=683.59, \quad P<0.001) \quad$ respectively. However, there was no significant relationship between the years of experience $(P=0.113)$ with the level of satisfaction in term of coping with stress.

Conclusion: Nurses perceived the professional work environment as somewhat satisfying, enjoying role in the unit and feeling the ability to reasonably handle the responsibilities of the role on the unit were the most satisfying aspects. Nurses' genders, clinical specialty, role in the unit were significantly correlated factors with the level of satisfaction in term of coping with stress. Healthcare institutions should develop strategies and guidelines that improve professional work environment and help nurses manage work-related stress.

Disclosure of Interest: None declared.

\section{NP005}

The Neglected Part - the Case Manager for Strengthening Care of Hematopoietic Stem Cell Transplantation Donors Y.-C. Huang ${ }^{1, *}$, C.-H. Tsai ${ }^{1}$, J.-H. Liu', J.-L. Tang ${ }^{1,2}$

${ }^{1}$ Tai Cheng Stem Cell Therapy Center, ${ }^{2}$ National Taiwan University Hospital and College of Medicine, National Taiwan University, Taipei, Taiwan, Province of China

Introduction: In hematopoietic stem cell transplantation, health providers and family members focus mainly on recipients needs. However, the care for donors, including mental support and consultation of drugs and physical symptoms, are often inadequate. It is also confusing for donors to receive different instructions from different sources. A case manager could serve as a coordinator to set up a 


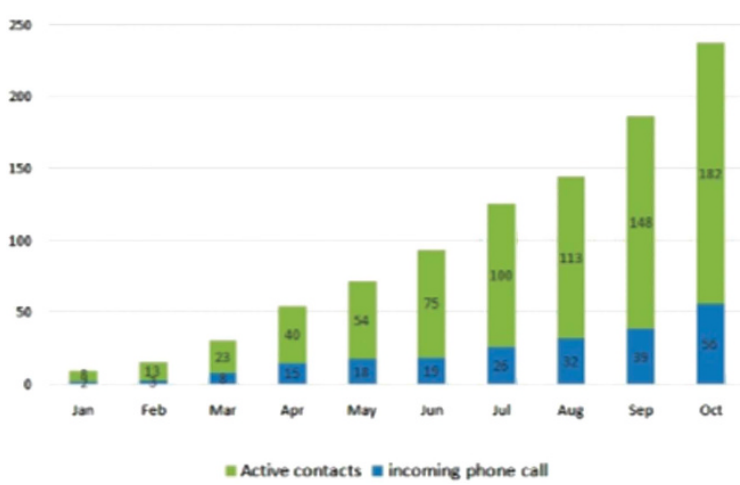

comprehensive care process. We share our experience regarding the case manager and the strengthening care path to improve the quality of care for the donors.

Method: From January to October in 2015, we set up a donor strengthening care path in our institute. First, we provided an individualized manual for each related donor. The manual described the donation process and schedule, nutritional education, wound care, and granulocyte-colony stimulating factor (G-CSF) instructions. Second, we arranged the case manager's visiting schedule for donors, either by active visit or phone call before donation, on the donation day, and one week after donation. Besides, additional incoming phone consultation was provided to every donor.

Results: Totally, there were $\mathbf{5 4}$ related donors handled by the case manager. The total donor contacts were 238 times, including 182 active visits $(\mathbf{7 6 . 5 \%})$ from the case manager to donors, and $\mathbf{5 6}$ incoming phone calls (23.5\%) from the donors to the manager. Before donation, there were 12 donors (22.2\%) having incoming consultations of drugs, even after educated by physicians at clinic. The consultations were mainly about G-CSF, and we found one donor misunderstood the dosage of G-CSF and successfully salvaged by the case manager. 13 donors (24\%) had incoming consultations about the blood sampling timing, items or relevant questions. 31 donors $(\mathbf{5 7 . 4 \% )}$ ) had incoming phone calls about the donation schedule or the catheter insertion process for donation. Among them, the schedule of two donors were missed by physicians but successfully arranged by the case manager. After Donation, 11 donors (20.3\%) complained about bruise over the catheter removal site, and $\mathbf{1 7}$ donors (31.4\%) had pain lasting over one week. 1 donor with catheter related cellulitis. They did not express the discomfort to physicians but to the case manager by the active phone contact one week after donation. The case manager successfully resolved all of their complaints, either by nursing education, proper mental support, or referral to the physicians. Conclusion: Our experience showed that the case manager was indispensable. Even after explanation, donors might still encounter questions, and the case manager could help them efficiently. By active visits, the case manager could help donors to deal with their unsaid discomfort, especially about the catheter for donation. On the basis of the 10-month donor strengthening caring experience, we could revise our high-quality care and nursing protocol.

Disclosure of Interest: None declared.

\section{NP006}

The development of a Nurse-Led Clincal Apheresis service in extra-corporeal photopheresis (ECP) for the treatment of small body weight patients

J. Guest ${ }^{1, *}, H$. Cheal ${ }^{1}$, J. Lawrence ${ }^{1}$, A. Gennery ${ }^{2}$, R. Skinner ${ }^{1}$

${ }^{1}$ Child and Adolescent Haematology/Oncology Stem Cell Transplant, ${ }^{2}$ Ward 3 HSCT unit, Great North Childrens Hospital Royal Victoria Infirmary, Newcastle-Upon-Tyne, United Kingdom
Introduction: Expansion of a Nurse-Led Clinical Apheresis service to develop a small body weight extra-corporeal photopheresis (ECP) service; the challenges of small body weight extra-corporeal photopheresis for graft versus host disease following allogeneic stem cell transplant within the paediatric setting.

Method: Small body weight ECP following haematopoietic stem cell transplant for the treatment of graft versus host disease is challenging. Establishing robust training and quality managment programmes to support the development of this service was integral in developing this successful small body weight programme.

Extracorporeal Photopheresis was commenced following failure of conventional treatment, with referral directly to the Paediatric Apheresis Nursing team. Immunosuppression was weaned as clinically indicated by our ECP Consultant and Medical teams.

Our small body weight programmes offers ECP with our smallest patient to date weighting $6 \mathrm{~kg}$. Quality of life improvements to children and their families has been key in developing a service that meets the needs of this complex patient group within the haematopoietic transplant specialty. Treatment was undertaken over varying time frames dependant on response to treatment, and following the initial inpatient treatment phases the procedures were undertaken on an outpatient basis.

Results: ECP within the small body weight group is challenging and part of the success of our service has been to optimise treatment programmes to enable us to have an overall impact on the morbidity and mortality of this specialist group.

Challenges in the small body weight population specifically include;

- Total blood volume and blood prime of disposable circuit

- Low flow/return rates

- Appropriate long term venous access devices to support apheresis

- Haemodymanic balance caused by extracorporeal circuit and buffy coat volume

- Line flushing and patency

- Anxiety and fear of the procedure

- Aniticoagulation - ACDA

- Morbidity and mortality associated with long term immunosuppression

- Environment and time on the machine

- Infections and cytokine release is the ECP working?

- User responsibility of machine/software

- Maintenace of appropriate haematocrit and haemoglobin levels

- Exposure to increased blood product support

Conclusion: Extracorporeal photophereis for small body weight patients following haematopoietic stem cell transplant who develop GvHD has been well-tolerated in our small body weight patient group when using blood-prime of the cellex photopheresis equipment, with resolution of GvHD possible.

Challenges for practitioners undertaking the procedures are described above. The development of our nurse led apheresis service to encompass ECP has been challenging, however the impact on our patient group has driven us to develop a robust quality driven service that is seen as a centre of excellence in small body weight procedures.

Disclosure of Interest: None declared.

\section{NP007}

The Development of a Care Bundle and Patient Care Diary to Facilitate Shared Care Across Multiple Care Settings for Patients Undergoing Extra Corporeal Photopheresis (ECP) A. Calderwood ${ }^{1, *}$, D. Potok ${ }^{1}$, J. Thornton ${ }^{1}$ on behalf of Care Bundles Group on behalf of Alison Thomas, Sarah Mills, Sarah Douglas

${ }^{1}$ Therapeutic Apheresis Services, NHS Blood and Transplant, Manchester, United Kingdom 
Introduction: Using clear methods of documentation, assessment, collaboration and first class care delivery, we can provide the best possible outcome for our patients. Care bundles and care diaries relating to an individual patient's treatment supports this ethos.

NHSBT provides ECP which is a second line treatment for patients with Chronic Graft Versus Host Disease (cGVHD) and Acute Graft Versus Host Disease (aGVHD) as well as Cutaneous T Cell Lymphoma (CTCL). Patients can expect to attend for treatments twice a week, every 1-2 weeks for several months to years. Interspersed with this, patients will have other ongoing treatments, follow up clinics and episodes of emergency admission to their referring clinical teams. Effective communication ensures the patient pathway is smooth, in particular communication with the referring teams promoting coordination of pre ECP requirements and treatment events There are many potential causes of non-attendance or delays to ECP treatment - for example, patient transport, inconvenient appointment times, conflicting or lack of coordination with other clinic visits etc. There are also stringent pre-requirements for ECP - hct, plt count, infection status, venous access etc., all which impact on the ability to perform the treatment and its effectiveness. Referring clinical teams frequently require updates around previous treatment events, treatment plans and interventions outside of routine follow up, and this information then has to be collated from various parts of the patient clinical notes. At times appointments have been missed, communication of requirements for treatment attendance not passed on to appropriate people or fully understood and details of treatment episodes not received by referring teams.

Method: To improve communication with patients, ECP practitioners and care givers, Therapeutic Apheresis Service (TAS) have produced an ECP care bundle and patient care diary to record, store and communicate information in a single document. The Care bundle contains

- Skin Mapping

- Nutritional status

- Ongoing treatment regimes

- Support agencies

- Vital Signs

- Infection status

- CVC monitoring

- Vascular device monitoring

- The care diary contains key information and ECP treatment requirements and is kept by the patient to share with referring, emergency and ancillary teams. It also contains overview informationabout the specifics of ECP requirements and post treatment expectations.

Results: An audit of customer and patient satisfaction surveys will show improvements in communication to and from ECP staff, patients, referring teams and support agencies.

Conclusion: Good communication between care givers and patients not only promotes a seamless service, but empowers patients to take control.

Disclosure of Interest: None declared.

\section{NP008}

Career track for qualified nurses in Haematology and Stem Cell Transplantation at the University Hospital Zurich (USZ)

C. Bischof ${ }^{1, *}$, J. Buder ${ }^{1}$

${ }^{7}$ Haematology, University Hospital Zurich, Zurich, Switzerland

Introduction: In world leading hospitals, the human resources development for qualified nurses on the basis of specific career models has proved of value. These hospitals were carefully selected with regards to skills shortage and were thus able to offer attractive career development options for nurses in order to prevent a brain drain. To further support the career options for qualified nurses at University Hospital Zurich, an eight-step career track was established. For each step, a specified job declaration was phrased to enable qualified nurses to develop their skills stepwise supported by specially trained tutors.

The USZ career track furthermore serves as a tool to assess the staff requirements and to standardize the fields of activity and responsibility in patient care.

Method: When beginning their work at USZ, every nurse will pass through a defined introductory teaching for six to twelve months. Afterwards, the participant undergoes a tightly organized set of learning modules for another six to twelve months. This basic program called 'Anniculum' is equivalent to step one and serves as the basis for a career at USZ. Each of the following steps further allow the participants to strengthen their theoretical and practical knowledge in patient care. In the Haematology and Stem Cell Transplantation (HSCT) unit, new nurses will be supervised by an experienced specialized nurse and will have three days per week set aside for further theoretical education. Within this education, basic aspects of hematologic malignancies are taught.

During step two of the career track, the nurse will then implement the newly built theoretical knowledge in complex practical patient care. This step, too, is supported by further training, with one element being an additional, broader haemato-oncological module. In these interdisciplinary modules, nurses as well as doctors report on haemato-oncological topics and cases to optimize patient care.

In order to be certified by the European Oncology Nursing Society (EONS), qualified nurses have to pass a test. This is the basis to take further steps in the USZ career track.

Results: The competencies for nurses specializing in haematological nursing shows the broad span of knowledge, skills and abilities required by a haematology nurse and demonstrates the complexity of the nursing care in haematology.

Conclusion: The establishment of the track may guide the individual nurse in professional development. The career track in Haematology not only enables nurses to gain insight into HSCT but also allows a for profound teaching in patient care. Disclosure of Interest: None declared.

\section{NP009}

What do allogeneic transplanted patients and nurses know about oral complications?

E. Cao ${ }^{1, *}$, B. Belegni ${ }^{1}$, S. Agazzi ${ }^{1}$, M. Cavalli ${ }^{1}$, C. Milanesi ${ }^{1}$, G. Pasini ${ }^{1}$, R. Greco' ${ }^{1}$,F. Giglio , F. Ciceri ${ }^{1}$, C. Soliman ${ }^{1}$

${ }^{1}$ Ematology and BMT Unit, IRCCS SAN RAFFAELE SCIENTIFIC INSTITUTE, Milano, Italy

Introduction: The aim of the survey is to verify the knowledge both of patients undergoing allogeneic hematopoietic stem cell transplantation (allo-HSCT) and nurses about the onset and the management of oral cavity complications caused by chemotherapy and radiotherapy. Moreover, to evaluate the importance of the dental hygienists roles in our Haematology and BMT Unit.

Method: The survey started in July 2015 and ended in September 2015. All 21 allo-HSCT patients and the 41 BMT Unit nurses were included. A multiple-choice questionnaire regarding the main topics were filled out from the nurses by themselves. For the patients, a dedicated nurse supported them for the questionnaire compiling during the hospitalization.

Results: We received back $81 \%$ of patients answers and $80 \%$ of completed questionnaires from the nurses. Most of the patients thought that dental hygenists are very important figures in the prevention and management of oral cavity complications, especially during the hospitalization. Moreover, according to the majority of patients involved, the most common problems at the hospital were mucositis and lack of appetite. The survey showed that there was an inappropriate patients knowledge about possible oral complications. The patients would like to receive more information about the correct behaviors and the techniques to alleviate pain and discomfort. Finally, the involved nurses wanted to acquire 
competence in the field of oral prevention and dental health service in order to manage complications and ensure the best care to allo-HSCT patients.

Conclusion: In our experience, the presence of different professional figures inside the helpful team represents a fundamental element to guarantee a complete and qualitative assistance to allo-HSCT patients. Both patients and nurses expressed the wish to became involved in some meetings with specialists in oral care to put into practice the recommendations during the patients hospitalization. In the future, we would have the objective to write specific recommendations available for patients and their caregivers during the hospitalization.

Disclosure of Interest: None declared.

\section{NP010}

Vaccination following stem cell transplantation in adults: initiatives to improve vaccination adherence and follow-up

J. De Munter, ${ }^{1, *}$, M. Quaghebeur ${ }^{1}$, T. Bauters ${ }^{2}$, I. Moors ${ }^{1}$, T. Kerre ${ }^{1}$ Hematology, ${ }^{2}$ Pharmacy, University Hospital Ghent, Gent, Belgium

Introduction: A systematic reimmunization is important to re-establish appropriate immunity in hematopoietic stem cell transplantation (HSCT) recipients and to decrease the risk of vaccine preventable infectious diseases with their related morbidity and mortality. Therefore, close follow-up of the vaccination status is important.

A previous survey performed at the Ghent University Hospital revealed that $76,5 \%(n=26)$ of the post allogeneic HSCT patients eligible for vaccination, were vaccinated on-schedule according to the in- hospital guidelines. In $8.8 \%$, a medical indication to delay the vaccination could be identified. Postponed vaccination without a medical reason was observed in a minority of patients (11.8\%). Following these observations, we explored possible initiatives to increase the vaccination rate in post HSCT patients.

Method: Literature data and specialized websites were analyzed for existing initiatives about quality improvement towards vaccination. Items as patient involvement, patient education and a multidisciplinary approach are known to result in an optimalization of the vaccination status. A preprogrammed vaccination calendar calculator is known to be a valuable tool.

Results: A preprogrammed vaccination calendar calculator is established for follow-up of all patients post-HSCT. The template contains a prescheduled agenda so that patients, their caregivers and the general practitioner have an overview of their proposed visits and upcoming vaccines.

At discharge, the physician consults the preprogrammed vaccination sheet directly from the electronic patient database and discusses the findings in an multidisciplinary approach. At the consultation with the nurse practitioner (day 100 post transplant), this sheet with data for vaccination is extensively explained to the patient and/or his caregiver. At that moment, the patient is directly involved and gets the necessary explanation and information about the vaccines. This educational step is an important part of the current standard care.

The patient can consult her/his own vaccination status and controls it through self-management.

Conclusion: Besides clear (inter)national post-transplant vaccination guidelines, the patient is directly involved at all moments about the importance of post-vaccination. A personalized follow-up vaccination sheet aims to decrease the number of postponed vaccinations without medical reason.

The multidisciplinary approach involving physicians, pharmacists and nurses, remains a key factor to increase vaccination rates. A follow-up survey, to show the effectiveness of these interventions, will be explored in the near future.

Disclosure of Interest: None declared.

\section{NP011}

Diagnostic significance of blood and removed central venous line cultures in patients with fever undergoing chemotherapy and stem cell transplantation

J. I. Pereligina ${ }^{1, *}$, O. Larisa ${ }^{7}$, P. Marina ${ }^{7}$, P. Olga ${ }^{7}$, M. Ivan ${ }^{7}$, D. Elena ${ }^{1}$, B. Maksim², A. Boris ${ }^{2}$

${ }^{1}$ Raisa Gorbacheva Institute of Children Oncology, Hematology and Transplantation, First Pavlov State Medical University of Saint-Petersburg, Russia, ${ }^{2}$ Raisa Gorbacheva Institute of Children Oncology, Hematology and Transplantation, First Pavlov State Medical University of Saint-Petersburg, Russia, Saint-Petersburg, Russian Federation

Introduction: In every patient with central venous line (CVL) and signs of infection without evidence of another localization, CVL is a potential source of infection. In neutropenic patients after chemotherapy (chemo) or hematopoietic stem cell transplantation (HSCT) infection is often a septicemia with severe intoxication when $\mathrm{CVL}$ - one of the entrance routs. Efficacy of blood cultures from CVL does not exceed $60 \%$ and depends on the blood volume collected, which is caused by the ability of microorganisms to form a biofilm. That is why the diagnostic significance of CVL culture might be higher than blood culture.

Method: The study included 33 patients with 42 infectious episodes (neutropenic fever without response to empirical antibiotics) after chemo and HSCT, in whom the CVL was removed. Patients were treated in HSCT unit between 01.01.2015 and 01.07.2015. The diagnostic significance was defined as percentage of positive results (identification of pathogen). The comparison was performed with Mann-Whitney test to determine the relationship between probability of positive result and frequency of blood cultures. Chi-square test was used to determine the optimal frequency of blood collections for a culture and compare diagnostic significance of blood and CVL cultures.

Results: In 42 infectious episodes when a CVL was removed the blood cultures were positive only in 10 cases, and overall diagnostic significance was $24 \%$ (blood cultures were drawn from 1 to 12 times, median 3). CVL cultures reveled the pathogen in 31 cases, overall diagnostic significance was $74 \%$. The efficacy of CVL cultures were 3 times greater. The calculation of efficacy of blood cultures according to number of draws, revealed that more than 4 collections resulted in $100 \%$ sensitivity when compared to less than 4 . But more than 3 blood culture collections were not statistically better than less (Chi-square $P=0.09$ ), which is probably caused by few observations.

Conclusion: In this study we observed that CVL culture is more effective method to determine the cause of fever than blood cultures in patients after chemo and HSCT. Probably, 4 or more blood draws for the culture is more effective than less. Further observations on larger number of patients are required.

Disclosure of Interest: None declared.

\section{NP012}

ESAS as a teaching tool for self-care in allogeneic stem cell transplantation

K. Holmberg ${ }^{1,2, *}$, K. Bergkvist ${ }^{3}$, C. Lundh Hagelin ${ }^{2}$

${ }^{1}$ Center for allogeneic stem cell transplantation, CAST, Karolinska University hospital, ${ }^{2}$ Sophiahemmet University Stockholm Sweden, Stockholm, Sweden, ${ }^{3}$ Sophiahemmet University, Sophiahemmet University Stockholm Sweden, Stockholm, Sweden

Introduction: Allogeneic hematopoetic stem cell transplanatation (allo-HSCT) is identified as one of the most stressful treatment methods in modern oncology where all the patients more or less will be affected physically and mentally. Because of the complexity of the treatment with the risk of complications and relapse of disease it is of great importance that the patient have the knowledge and the skills to exercise self-care. Dorothea Orem believes in her theory of nursing that 
the nurse should as an educator help and support the patient so she can exercise self-care and thus be as independent as possible during and after severe illness.

The aim of the study was to describe patient's perceptions of knowledge acqusition about their health and self-care with support of a subjective symptom assessment instrument (ESAS) in allogeneic stem cell transplantation.

Method: An empirical qualitative interview study was conducted with six informants who performed symptom assessement with an subjective symptom assessment instrument, ESAS (Edmonton symptom assessment system) during hospital stay. Data collection was analyzed according to phenomenographical method.

Results: The preliminary results showed that the conversation between patient and nurse after the symptom estimate was made were important so the instrument would fill the knowledge acqusition of the informants. When the nurses had the following conversation about the estimated results the informants expressed that ESAS could bring order among different symptoms and feelings. Some of the informants used self reflection in the context of self-assessment. The informants also expressed a need for a subjective self-assessment instrument in the outpatient setting. All informants practiced self-care that included more than drug management and responsibility for medical appointments. Daily all informants performed some form of self-care, mainly in the form of examination of physical health status.

Conclusion: The mutual communication and reflection between the patient and the nurse can capture knowledge about their own health promoted by a subjective selfassassment instrument, ESAS. When patient's knowledge about their own health became deeper this had a positive impact on the practice of self-care both on the ward and in the home.

Disclosure of Interest: None declared.

NP013

Implementation of Ambulatory Care treatment pathways for Acute Leukaemias at Hammersmith Hospital - Staff's Perspective

N. Rizvi ${ }^{1, *}$, O. Brokka ${ }^{1}$, F. Sarkari ${ }^{1}$

${ }^{1}$ Haematology Outpatient, IMPERIAL COLLEGE HEALTHCARE NHS TRUST, LONDON, United Kingdom

Introduction: Treatment of acute adult Leukaemia typically consists of intensive induction followed by high dose consolidation chemotherapy as an in-patient as has been the case in the clinical haematology department of Hammersmith Hospital.

We will explain how moving a long-standing intensive chemotherapy regimen for acute leukaemia to the outpatient setting is safe, feasible and improves the patient experience, but main focus will be nurses' perspective on caring for patients on an Ambulatory Care (AC) treatment pathway for acute leukaemia.

Method: A multidisciplinary team was involved in developing patient pathways for Ambulatory Care which included doctors, nurses, nurse managers, general managers and pharmacy. Clear cut guidelines were developed for chemotherapy administration, patient education and nursing roles and responsibilities.

The haematology unit formally began its ambulatory care programme for acute Leauamias at the beginning of 2015.with patients attending the Haematology Day Care unit once or twice a day depending on the regimen.

Although most of the nursing staff in the Day Care unit have a good understanding of the haematology patients' malignancies and the patients' journey, not everyone had experience in treating acute Leukamias. The unit introduced internal rotation (to in-patients wards) in 2013 (Logan, 2014) and this has helped improve nurses familiarity with the various assessment tools used to monitor the patients. However to ensure high quality and safe care all nurses were educated to assess patients on daily basis for the side effects of chemotherapy; such as oral assessment for mucositis, nutrition intake, fluid intake, weight monitoring, assessing for diarrhoea, vomiting and sepsis. A doctor is always available should the nursing assessment reveal any abnormal findings.

Results: Patients were educated to contact 24 hour helpline if they feel unwell or are having fever. A ring fenced bed was allocated for patients to be admitted into a haematology ward if they became unwell and required admission.

At the beginning of the AC treatment pathways programme some nurses were apprehensive and reluctant to learn. However, as they were educated regarding care pathways and observed colleagues treating patients they became more confident. Currently all chemotherapy certified staff are engaged in treating acute Leukaemia patients on the ambulatory care pathway.

Conclusion: Senior nurses will conduct an evaluation of the process including nurses' feedback from the implementation to learn what could have been done differently to prepare the nursing staff for this change. This will be valuable as the next

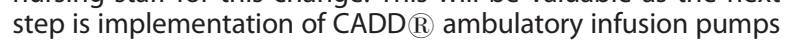
to be used for evening doses of chemotherapy and for the treatment of Lymphoma patients on an Ambulatory Care basis.

Disclosure of Interest: None declared.

\section{NP014}

The implementation of an E-Journal Club on the Haematology and BMT unit in Bristol

R. Hallam ${ }^{7}$, A. Smith, ${ }^{1, *}$

${ }^{1}$ Haematology, Bristol Haematology and Oncology Centre, Bristol, United Kingdom

Introduction: In 2014 the BMT and haematology units at the University Hospitals NHS Foundation Trust combined and a new purpose built ward was created to care for all haematology and transplant patients.

It was identified at a very early point in the amalgamation that the BMT nurses and the haematology nurses each had their own individual nursing skills and knowledge base. The challenge would be to ensure all the nurses on the unit had appropriate access to education so that the BMT nurses were competent and felt safe and skilled in caring for haematology patients and the haematology nurses were appropriately trained to care for transplant patients.

The Haematology Clinical Nurses Specialists and Education Sister decided that a Journal club, alongside formal education sessions, would be an effective learning tool to assist in providing up to date, quality education to a large group of nurses with a variety of clinical skills and experience.

Method: The literature recognises that a journal club facilitates discussion and evaluation of new research and considers the application to practice and improvement in patient care. The advantages of using a journal club are that members of the group can update knowledge, learn to evaluate the strength of the evidence, promote implementation of new knowledge into practice and improve patient outcomes.

With over registered 50 nurses to include within the education sessions, a formal meeting would not be practical and so the idea of an E- Journal Club was formulated. The essence of the E- Journal club was that relevant journal articles would be emailed to all members of the group and discussions on the articles would be held via an on line platform as well as at weekly, informal ward based sessions.

Results: The initial E- Journal club was for all staff nurses on the ward. A review of the programme using staff feedback was undertaken 4 months later and the E-Journal club was reported to be a useful, interactive and educational resource. The feedback also identified that the health care assistants were also keen to be involved in the E- Journal club and had their own educational needs. At this point the E- Journal club 
was extended to include all members of staff on the haematology and transplant unit.

Conclusion: The E-Journal club has shown to be an effective and useful educational tool for informing, updating and facilitating learning for staff nurses and health care assistants on a busy haematology and BMT unit. The E- Journal club has prompted discussion about nursing change, reacted in a timely manner to ward nursing issues and been a wellreceived resource to deliver effective and appropriate education.

Disclosure of Interest: None declared.

\section{NP015}

Determination of Prior Educational Needs of Bone Marrow Transplantation Recipients in Our Society: Multicenter Qualitative Study

S. ERDAL 1,*, G. BOZ ${ }^{1}$, S. KOCUBABA ${ }^{2}$, S. BOSTANCl ${ }^{3}$, F. BILGIN ${ }^{4}$, R. OREN ${ }^{5}$, H. S. BEKOZ

${ }^{1} B M T$ Unit, Medipol Mega University Hospital, Istanbul, ${ }^{2} B M T$ Unit, Dr. Abdurrahman Yurtaslan Oncology Hospital, Ankara, ${ }^{3} B M T$ Unit, Inonu University Hospital, Malatya, ${ }^{4} B M T$ Unit, I.U. Capa Medicine Faculty, Istanbul, ${ }^{5}$ BMT Unit, Akdeniz Medicine Faculty, Antalya, Turkey

Introduction: The education of the patient and family members is a well known method to understand the diagnosis and treatment correctly, that facilitates active participation in their own care and resolution of "the feelings of desperation" associated with the disease. This period includes not only the duration of inpatient care, it is also an active process in outpatient setting after discharge. For this reason, information of our patients starts, in the beginning of hematopoetic stem cell transplantation (HSCT) both written and verbally in our units. However the observation of the repetitive questions in the same subject of some patients may suggest that some patients do not benefit in expected level from these information procedures. Our aim; this study was conducted to identify the prior educational needs and determine the most appropriate health education service in patients who were hospitalised for HSCT; by revealing perceptions about HSCT and considering the structure of our society.

Method: One hundred patients that HSCT was performed between May 2015-July 2015 in five HSCT centers operating in four different regions of Turkey included in the study. In the first day of HSCT unit, the patients were given a blank piece of paper and asked to write the questions that they wonder until discharge. Collected questions were grouped under headings: HSCT procedure, Hygiene, Communication, Body image, Social Life, Nutrition, Isolation, Sexuality, Religion and Discharge. Data were analysed into first asked 10 questions and most frequently asked 10 questions. Results reported as numbers and percentages.

Results: Of 100 patients enrolled in the study 81 have written at least one question, 4 did not write any question and 15 patient have written the expression of their feelings instead of questions. The most frequent written expression of the feelings were the word "Scared (Korkuyorum in Turkish)". Of 58 different questions, the questions about concerns of life like "What problems occur during the transplantion?", "Will I be okay?", "How many days will I stay?" were in first place. When all questions considered in general, the questions of hygiene were at a lower place and there was no question about religion and sexuality.

\section{Conclusion:}

In this study we determined that bone marrow transplantation recipients have anxiety, mostly focus on issues related to their health as expected. For this reason, initial evaluation of anxiety level, with proceeding education, based on the identification of the prior needs is important for more comfortable transplantation period and more effective education for HSCT recipients. In this context, a pilot study has been conducted in participating centers in this study.

Disclosure of Interest: None declared.

\section{NP016}

THE FIRST HEMATOPOIETIC CELL TRANSPLANTATION (HCT) ACTIVITY IN UZBEKISTAN AND IMPORTANT ROLE OF TRAINING IN HCT NURSING

S. Kocubaba ${ }^{1, *}$, G. Adibelli ${ }^{1}$, K. Citlak ${ }^{1}$, A. Kilinc ${ }^{1}$, O. Kayikci ${ }^{1}$, A. I. E. Tekgunduz ${ }^{1}$, F. Altuntas

${ }^{1}$ HSCT Clinic, Ankara Oncology Education and Research Hospital, Ankara, Turkey

Introduction: HCT activity is an important parameter defining developmental stage of a given country.There is a continuing increase in HCT rates in developed countries. But in certain parts of the world, HCT activities are yet to begin. In cooperation between Turkish Cooperation and Coordination Agency(TIKA) and Uzbekistan Ministry of Health, in the context of the Cooperation Project on Hematology and Bone Marrow Transplantation, the first HCT center was established in 2011 in Tashkent. Upon the request by Uzbekistan Ministry of Health and in cooperation with the Turkish Ministry of Health, a training program was prepared for Uzbek physicians and nurses. We aimed to report the educational training of Uzbek nurses in Turkey as part of the aforementioned project and share the preliminary activity of the first HCT center in Tashkent-Uzbekistan.

Method: The demographic, educational level, the professional experience of nurses, who were involved on the project were summarized. We also reported an overview on educational activities in HCT nursing.

Results: In 2012-2013 period a total of 6 nurses (median age: 40; range: 30-46) received training at Ankara Oncology Training and Research Hospital for 6 months in the area of HCT. Median professional experience of nurses were 20 (9-26) and 15 (2-26) years in general and oncology nursing practice, respectively. All participants involved in daily theoretical courses for 2 hours. The theoretical course included all relevant topics on HCT like standard definitions and terminology, indications, general nursing practice, supportive care, febrile neutropenia, mobilization, follow-up of patients for early and late complications of HCT. On the rest of a working day, nurses worked as observers in HCT clinic under supervision of experienced HCT nurses. The main problems of Uzbek nurses were communication with patients/colleagues and different standards in nursing practice between countries. As all Uzbek and 2 Turkish nurses spoke Russian language, Russian was the main spoken language through the program. HCT activity in Tashkent begun on March 2014. As of May 2015, 10 myeloma patients successfully received autologous HCT in Uzbekistan, who were served by participating Uzbek nurses of this project. After median 14 months of follow-up no patient experienced transplant-related mortality.

Conclusion: The Cooperation Project achieved its purpose by establishing the first HCT center and even so by performing first HCT activities in Uzbekistan. Great support by organizations and incredible efforts of HCT physicians, nurses and technical staff in both countries, were major drivers of this successfull project. We believe, that through rigorous education and close collaboration between partners, more patients in need can benefit from HCT worldwide.

Disclosure of Interest: None declared.

\section{NP017 \\ MIRROR CONVERSATIONS AS AN INSTRUMENT TO IMPROVE QUALITY OF CARE IN PEDIATRIC HEMATOPOETIC STEM CELL TRANSPLANTATION}

T. Neuman-Van Eijk ${ }^{1, *}$, L. Ball', C. De Vogel ${ }^{2}$

${ }^{1}$ WAKZ, ambulante zorg, 2 advisor healthcare logisitics, LEIDEN UNIVERSITY MEDICAL CENTER, Leiden, Netherlands

Introduction: In the Netherlands quality of healthcare (QOC) is an important issue. As healthcare providers we often assume to know what is the best for our patients and make decisions based on our expertise initiating new procedures, directives and protocols. However, the best referent of the quality of care 
is the patient, a viewpoint that has become increasingly important in our existing healthcare system. To explore patients experiences concerning QOC, we need a novel approach, incorporating the patients viewpoint.

Aim: To ascertain the experiences of children and parents undergoing hematopoietic stem cell transplantation (HSCT) by the use of 'mirror conversations'. and to learn from their experiences by active listening in order to improve the QOC offered in our unit.

Method: Introducing structured 'mirror conversations' with trained professionals led to exploratory and narrative conversations. The interlocutors were the patient themselves. They told their stories and experiences of their time in our hospital, both as in patients undergoing SCT or as outpatients at follow-up. There were no subjects planned in advance. The professional leaders were specially trained hospital employees who facilitated and guided the conversations. Prior to this they interviewed the organizing staff requesting the mirror conversation. The organizers gathered questions concerning what the workers wanted to know from their patients. These themes could be used in the conversations.

During the mirror conversation hospital staff were present in the audience but were not allowed to take part in the conversation, other than to listen to the patient stories. The patients defined the subjects in the conversation and talked about their experiences.

The conversations were taped to get an accurate report. On the base of this report a SWOT analysis was made. Areas of improvement were defined and subsequently addressed.

Results: The results of this conversation in a group of young HSCT patients and their parents was analysed.

The patients, parents and the workers of the hospital who participated were very positive about this experience. Significantly a number of standard procedures have been adapted as a result of this intervention.

Conclusion: Mirror conversations contributes to the quality of our professional care in pediatric HSCT. The patient perspective is made clear by the procedure of the mirror conversations.

Disclosure of Interest: None declared.

\section{NP018}

Standardising admission information for Paediatric Stem Cell Transplant patients

V. Cassels ${ }^{1, *}$, E. Scott ${ }^{1}$

${ }^{1}$ University Hospital Bristol, Bristol, United Kingdom

Introduction: Following a recent transition from a single Stem Cell Transplant (SCT) unit to a combined oncology ward, admission information given to SCT patients and their families has become inconsistent due to a newly formed team and significant junior nurse recruitment. The aim of this study is to educate nurses and other members of the multidisciplinary team (MDT) through the development of a tool, which will standardise information given during admission.

Method: A questionnaire was developed to establish current awareness and understanding of admission information. From the data attained and further consultation with the MDT, both within our unit and other trusts, an admission tool was constructed and approved. The tool was disseminated across the MDT to allow for a unified understanding of admission information, to ensure consistency within the team when educating patients and their families. Following inplementation, a questionnaire was used to evaluate and gauge the admission tool's effectiveness.

Results: There was clear evidence of knowledge gaps and uncertainties across the MDT regarding individuals' awareness of admission information. Since implementing this tool, the MDT's confidence and understanding of admission information has significantly improved, this is reflected in the post-education questionnaire conducted.

Conclusion: From highlighting gaps in the MDT's knowledge, and following their involvement, a tool has been created to standardise information given during admission. This has successfully contributed to positive reinforcement of knowledge and understanding, subsequently leading to a unified approach of a SCT admission. Cascading this information to new members of the MDT needs to be carried out to ensure uniformity with all members of staff to prevent any future confusion in patient and family education.

Disclosure of Interest: None declared.

\section{NP019}

Central venous catheter options for autologous stem cell transplants-a retrospective audit to inform how best to PICC?

C. Hawkins ${ }^{1, *}$, U. Vithanarachchi ${ }^{1}$, R. Salim ${ }^{1}$

${ }^{1}$ Haematology, Royal Liverpool \& Broadgreen University Hospital Trust, Liverpool, United Kingdom

Introduction: In keeping with other stem cell transplant units our institution is experiencing an increase in our autologous stem cell transplant workload. Due to the resulting pressure on resources and the perceived advantages for patients there has been a recent shift towards using Peripherally Inserted Central Catheters (PICCS) for some autograft patients. There are a number of publications supporting this practice and highlighting likely benefits including speed of insertion, reduced complication rates and reduced use of resources. In response to some practical difficulties experienced in individual cases we undertook a local audit to review our local experience with PICC lines in the autograft setting.

Method: We undertook a retrospective audit over a 6 month period. All autograft patients from our unit during that time were included and data was collected on the type of line used, any specific reasons as to why a particular line was preferred for that individual, the length of time that the line remained in situ, the reason for line removal and any complications experienced. Feedback was also collected from nursing staff and patients about their experiences.

Results: A total of 30 patients were included in the audit and these were distributed roughly equally between PICCs and Hickman lines. Overall complication rates were similar for both types of line and, although PICCs are less resource intensive in terms of insertion, this was to some degree off-set by a higher rate of occlusion requiring further intervention. In addition there were examples of patients with PICC lines requiring additional canulation for stem cell infusion. However, some clear factors were identified which could be used to aid selection of the most suitable line for individual patients.

Conclusion: PICC lines offer a viable alternative to Hickman lines for autologous stem cell transplant patients. They can facilitate cost-savings in terms of insertion and length of stay and have similar complication rates. However, problems can arise from higher occlusion rates and sometimes additional IV access is required. Consequently it is our belief that PICC lines can have a role in the autologous stem cell transplant setting but that they should be reserved for carefully selected patients.

Disclosure of Interest: None declared.

\section{NP020}

"Outcomes of epidemiological and management complications in the use of Picc catheter in hematology" F. Salvatori ${ }^{1, *}$, C. coratella ${ }^{1}$, C. tritini ${ }^{1}$

${ }^{1}$ haemathology, San Camillo Forlanini Rome, Rome, Italy

Introduction: Observing an increase of use of Picc catheters in our medical unit, we will describe the complications verified focusing on the appropriateness of their removal and the customs used in hematological division and bone marrow transplant unit of the San Camillo - Forlanini hospital of Rome. Method: It was conducted a retrospective study, from May 2012 to September 2014, on all of the adult patients subjected to a Picc catheter implantation, either in regime of 


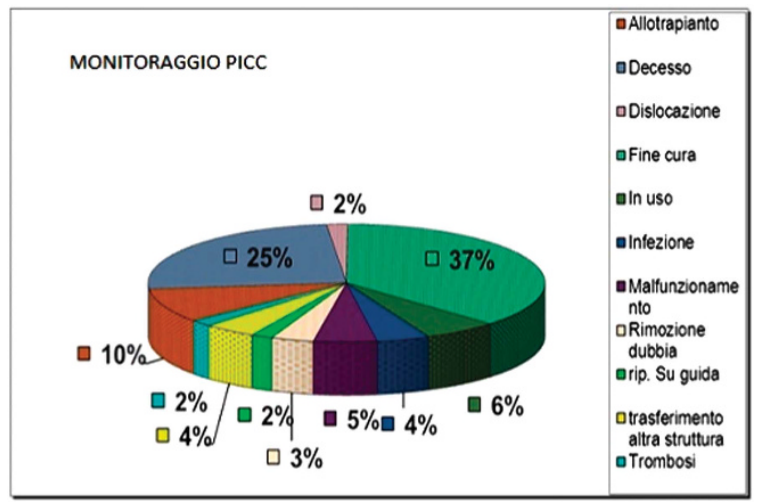

hospitalization or in Day Hospital. The epidemiological studies were conducted together with the Microbiological Service of the hospital.

Results: 131 Picc catheters were implanted and 116 were analyzed; the data of 15 were lost during the follow up. All of the devices have been followed till their removal the rate of infection found was of $6 \%(7 / 116)$, two of these removed for decease. From the results of the study, the other complications were: thrombosis $2 \%$ (2); malfunction $5 \%(6)$; accidental removals $2 \%(2)$; repositioning $2 \%(2)$; removal on evidence of only fever $3 \%(4)$.

The overall rate of complications have been verified in $20 \%$ of the cases (23), $10 \%$ of these were supposedly removed improperly.

The rest of the Picc catheters have been removed for decease in $25 \%$ of the cases (29), for end of the therapy $37 \%(43)$ and for transfer in other hospital $4 \%$ of the cases (5). Another $6 \%$ (7) had still the catheter in use. Finally, 11\% (11) had been removed to be replaced with another central venous access because in our operational transplantation unit either bone marrow or stem cells are infused with Hickman Catheter.

Conclusion: The most critical issue is the management of PICC catheters: of all the 116 analyzed, the $10 \%$ (12) were removed for complications sometimes premature. These added to the cases of removals for transplantation (due to use in our unit) $10 \%$ (11) mean that improper removal cases (due to unknowing of the devices characters and management) are still too high as data evidenced in scientific the articles found in matter.

The PICC catheters are a simple alternative to the traditional cvc devices, but the rate of complications is elevated and could be decreased by a strict training. There has been presented a protocol on use and management and in planning a training course about these problems. There is in course a prospective study to verify if there are improvements regard; the data will be presented in seat conference.

Disclosure of Interest: None declared.

NP021

How much is really enough? Increased Stem Cell target doses and the implications for the Apheresis Service J. Baker ${ }^{1, *}$

'haemato-oncology, ROYAL MARSDEN HOSPITAL NHS TRUST, Sutton, United Kingdom

Introduction: Autologous stem cell transplant remains the standard of care for Myeloma, and is dependent on the collection of a sufficient number of CD34+ cells. It has long been acknowledged that the minimum CD34+ dose for a single autologous transplant is $2.0 \mathrm{x}^{10 / 6} \mathrm{CD} 34+$ cells $/ \mathrm{kg}$. However, many transplant centres and guidelines are now advocating much higher doses $\left(2.5-5 x^{10 / 6} \mathrm{CD} 34+\right.$ cells $\left./ \mathrm{kg}\right)$. Indeed current practice is to collect enough cells for cryopreservation for a potential future second transplant, therefore collection doses may be targeted at $>/=5.0$. This clearly impacts on patients and Apheresis nurses, and the processing and storage services.

Method: An audit was performed on all Myeloma mobilisation episodes for the past 12 months to assess how many patients had reached their ideal target dose or the minimum accepted stem cell dose. It was then determined how many additional Apheresis episodes would be required for patients to reach the new higher target doses advocated, or indeed if these patients would be capable of mobilising the significantly greater CD34 cells without intervention with Plerixafor.

Results: From September 2014 - September 2015, 76 myeloma patients completed a mobilisation regimen. The majority 68 patients $(90 \%)$ completed a Chemo-G mobilisation, and 16 patients $(21 \%)$ received Plerixafor. 63 patients $(83 \%)$ collected the minimum stem cell dose $\left(2.0 \times{ }^{10 / 6}\right.$ CD34+ cells $/ \mathrm{kg}$ ) for 1 auto and 46 patients (61\%) completed this with 1 Apheresis episode. If the new stem cell target guidelines were implemented it can be estimated that $15(20 \%)$ patients would require a 2 nd Apheresis episode and 4 patients (5\%) would required a third day. Additionally $4(5 \%)$ would require a second dose of Plerixafor and a further $5(7 \%)$ would require intervention with Plerixafor.

Additional issues:

- Robust audits of engraftment data on lower versus higher stem cell doses

- Audits of long term outcomes with increased infusion doses - Larger volume leukopheresis and implications associated with this

- Larger economic healthcare impact regarding increased use of high cost drug-Plerixafor

- Discussion of necessity to collect enough stem cells for 2 auto's for all myeloma patients,i.e. prognostic factors, age limits etc

- Calculating stem cell dose on ideal body weight as opposed to actual therefore allowing smaller infusion doses

Scheduling and 7 day week working

Conclusion: Stem cell collection, processing, and storage is a resource intensive service and will be exacerbated as guidelines are implemented requiring increased CD34+ cell target doses for Myeloma patients planned for 2 potential autologous transplants. Apheresis nurses are pivotal in optimising Apheresis procedures, utilising Plerixafor appropriately, scheduling efficiently, and participating in audits that will promote evidence-based practice.

Disclosure of Interest: None declared.

\section{NP022}

Improving multi-disciplinary team communication and patient management with the 'Daily Haematology Consultant Discussion' sticker

M. Waller ${ }^{1, *}$

${ }^{1}$ MANCHESTER ROYAL INFIRMARY, CENTRAL MANCHESTER NHS FOUNDATION TRUST, Manchester, United Kingdom

Introduction: Patients undergoing haematopoietic stem cell transplant often have multiple problems which require input from various members of the multidisciplinary team. Increasing numbers of haematology patients has additionally led to growing pressures on inpatient beds. As such a twice daily Consultant board round discussion was implemented to ensure senior input in patient management and clinical decision making. The consultant board round was initiated following the Trust wide 'perfect week' campaign which was an initiative to try and optimise patient care, identify treatment or investigation delays and expedite the discharge pathway. This initiative was embraced in an attempt to break the 'cycle' and improve efficiency in the department, results were published by NHS England (NHS England, 2015). This created an opportunity to identify patients who were suitable for early supported discharge who could attend the haematology day unit for ambulatory care.The attending Consultant held a "board round discussion" with the multidisciplinary team which included medical staff, advanced 
nurse practitioner, nurse in charge and pharmacist twice a day at nine am and five $\mathrm{pm}$. It became apparent that the consultant discussions were not being documented in the patient's case notes in real time despite the fact that changes to treatment pathways were suggested or agreed. Record keeping is an important part of effective patient care as it provides evidence of specific situations, decisions and actions (NHS England, 2014). In order to address this a sticker was designed- these stickers are user friendly \& easy to apply in the medical notes as evidence that a discussion has taken place. Method: A documentation sticker was created to ensure daily inpatient team discussions were documented in patients' medical notes changes to care \& treatment plans communicated antibiotic \& antifungal therapy was accurately documented to improve compliance with antibiotic audit outstanding test or results could be expedited issues delaying discharge addressed to improve admission \& discharge process patients suitable for early discharge could be identified.

Results: - sticker successfully implemented as evidence of Consultant discussion with MDT

- Stickers easy to complete as discussions takes place

- documented in real time and fixed in patients case notes

- intravenous antibiotics and anti fungal therapy documented to comply with antibiotic audit

- Issues affecting discharge considered and addressed in a timely fashion -improving discharge

Conclusion: The development and implementation of the 'Daily Haematology Consultant Discussion sticker' has proved to be an effective tool that is easy to use and fix in patients medical notes. The data captured on the sticker has improved communication within the multi-disciplinary team and patient care.

Disclosure of Interest: None declared.

NP023

A review of the nutritional management of patient's diagnosed with gut GvHD post bone marrow transplantation

N. Scott ${ }^{1, *}$, A. Burton ${ }^{1}$

${ }^{1}$ Nutrition and Dietetics, St James's University Hospital, leeds, United Kingdom

Introduction: Our aim is to review our units' nutritional management against recent recommended guidelines. A review by Van der Meij et al (2013) looked at the evidence around for nutritional support in patients with gut GvHD. Following critical analyse of the data available the authors went onto develop recommendations for nutritional support. Method: This was a retrospective review 2008-2015 using dietetic record cards. The data obtained was: stool volume on admission, feeding route used to provide nutrition on admission and throughout their hospital stay. In addition admission weight was collected allowing \% weight loss to be calculated using post transplant discharge weight.

Results: In total 17 patients were referred. Nutritional intervention was provided to 16 out of the 17 patients. One patient died before a nutritional plan could be provided. All 17 patients had stool volumes of $>500 \mathrm{mls} /$ day on admission (ranging from $500->3000 \mathrm{mls} /$ day). From the 16 patients with nutritional plans 10 patients $(62.5 \%)$ had parenteral nutrition (PN) as their first line nutrition. Eight of these 10 patients (80\%) went onto oral diet or enteral feeding (EF) once their stool volumes were $<500 \mathrm{mls} /$ day. In the 6 patients who did not receive $\mathrm{PN}$ initially there was an equal split between oral diet and EF as their first line nutrition. From this group of 6 patients, 2 needed to switch to PN due to stool output remaining $>500 \mathrm{mls} /$ day.

Conclusion: The recommendations state patients with severe diarrhoea $>500 \mathrm{mls} /$ day should receive PN. Therefore indicating that all our 17 patients should have received PN. The results show that $56 \%$ of the patients had lost clinically significant amounts of weight ( $>10 \%)$ on admission.

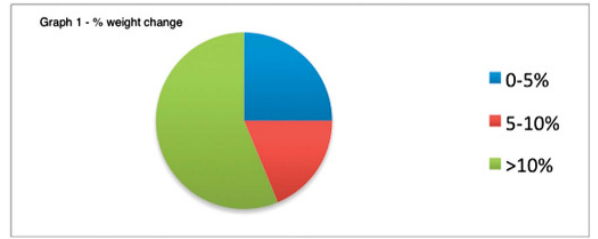

Which could indicate an argument for aggressive nutritional support from the start in a form that the patient would absorb, which PN would provide. What this review of our management does show is we are waiting for the stool output to be $<500 \mathrm{mls}$ before considering switching from PN to either EF or oral diet. Which is what the recommendations indicate. The authors however do not give guidance on when to use the enteral or oral route. One point we must consider is we are reviewing our practice from 7 years ago and our philosophy has been to use the gut first if it can be accessed. However this may no longer be the most appropriate in this complex group. Therefore now we need to work alongside the medical team to implement these current recommendations in the initially phase once a diagnosis of gut GvHD and the stool volumes are $>500 \mathrm{mls} /$ day.

Disclosure of Interest: None declared.

\section{NP024}

Developing nurses' competences as a focused and interconnected effort in a transplant unit

T. B. Sørensen ${ }^{1, *}$, M. M. Nielsen ${ }^{1}$

${ }^{1}$ Hematology, Aarhus University Hospital, Aarhus, Denmark

Introduction: In 2014 a number of nurses did not feel qualified to undertake the complex transplant nursing. They had a low job-satisfaction and the cooperation with one another and the physicians could be better. A training program had been drawn up, but was not effective. As a part of an upcoming JACIE accreditation, the qualifications and training of nurses had to be documented. It all motivated us to start solving the problems.

Method: Aim: All nurses should acquire the necessary competences, and become able to manage the professional demands.

Against the background of the theories on developing nurses' qualifications (Kirkevold), on well-being (Jensen \& Johnsen) and on interdisciplinary cooperation (Lauvås \& Lauvås), actions were taken. The SOP for the training program was adjusted so that it included the following for newly employed nurses:

- They were all assigned a preceptor for half a year

- time for training and reading SOP's was scheduled

- transplant related lessons were introduced

- interviews after 1, 2, 3 and 6 months and a 6 months deadline for completion of the training programme and documentation of core competencies were introduced

- substitutes were offered the same training as permanently employed.

To ensure a continued development of all nurses competencies:

- Primary nursing was substituted with partnerships

- daily nursing conferences were introduced

- weekly lessons for all nurses were introduced

- an annual scheduled training day focused on care of transplant patients was planned

- all nurses attend an individual annual staff development interview to evaluate their competences and job satisfaction, and an individual action plan is drawn up.

An online documentation system for qualifications, training and competences was implemented for all nurses and an online anonymous interview was used to evaluate various areas concerning job-satisfaction.

Results: The program has allowed cooperation, discussions and feedback, which have contri-buted to learning and 
improved the professional identity. The nurses can distinguish better between nursing and medical problems and possess a higher level of know-ledge which has qualified the cooperation with the physicians. Experienced nurses are visibly focused on developing the nursing, to teach colleagues and to devise nursing guidelines. The partnerships have given a more even division of work and have in-creased the adaptability. Inquiries on the psychological working environment and the annual staff development interviews have shown that the job satisfaction has improved significantly.

Conclusion: When training and development of competences are brought and kept into focus through the organisation of the unit, it can improve the general professional level. It is distinct that the time consumption spent on training and professional considerations has been worth while, because the nurses are feeling safer and are working more steadily and independently than previously.

Disclosure of Interest: None declared.

\section{NP025}

Nursing Manpower Allocation Based-on Working Hour Measurement of Bone Marrow Transplant Unit in China

Z. Yang ${ }^{1, *}, X . Y^{1}{ }^{1}, X . X u^{1}$ on behalf of He Xin-xin, Sun Hui, Liu Xiao-lin, Li Dan, Yi Xiang-li, Wang Yu

${ }^{1}$ Institute of Hematology, Peking University, Beijing, China

Introduction: Reasonable allocation, utilization and development of nursing human resource is the basis to ensure the sustainable development of the nursing career. Allocating manpower according to the ward nursing workload is an important nursing human resource management method. And working hour measurement has been commonly used to determine the nursing workload.

Nursing work load in different wards and areas is often affected by various factors such as disease status and bed turnover. Influenced by the professional particularity, the daily nursing procedures and basic nursing items in bone marrow transplant unit are significantly different with that in general care unit. The common ward manpower allocation and nursing-to-patient ratio can not be used directly. This study aims to determine the bone marrow transplantation unit nursing workload through working hour measurement, and explore the proper nursing workforce allocation.

Method: Time frame: from 14th December 2014 to 28th December; Site: a bone marrow transplant unit with 23 beds of a general hospital which is affiliated to a university. All nursing items related to the patients during this time frame are being measured, including direct and indirect nursing items.

During the process, the level of nursing, job position, working hours, starting and ending time of each nursing items are being observed by a team of trained observers.

The record data is then analyzed and the total frequency and working hour of each nursing item is calculated after the measurement. Then the mean frequency and working hour for one patient per day is also got. At last, the nurse workforce in need can be calculated.

Results: The unit involves 95 nursing procedures, including 65 direct care procedures and 30 indirect nursing procedures. Direct nursing care contains observation and assessment, therapeutic care, life support care, symptoms management, patients education, charge/discharge management. Indirect nursing care contains record and file related works, preparation of instruments and articles, information checking/ confirming. On average, the total working hour of each patient in 24 hours is $\mathbf{7 3 0 . 8 6}$ minutes. The theoretical ratio for patient to nurse is $1: 2.3$.

Conclusion: Nursing care in bone marrow transplantation unit has its specialty. Working hour measurement on the basis of daily situation is a scientific method to explore the clinical nursing workload and determine nursing manpower allocation.

Disclosure of Interest: None declared.

\section{NP026}

Introducing a screening tool for patients attending a Photopheresis Unit

C. Rushton ${ }^{1}$, R. Goodgrove ${ }^{1, *}$, M. Foster ${ }^{1}$, T. Maher ${ }^{1}$, L. Robertson ${ }^{1}$, A. Alfred ${ }^{1}, P$. Taylor ${ }^{7}$

${ }^{1}$ ROTHERHAM HOSPITAL, Rotherham, United Kingdom

Introduction: Demand for ECP (Photopheresis) has increased and patients conditions vary it is important to establish which patients are at risk of developing a pressure sore. Patient groups presenting for ECP are at risk of developing a pressure sores due to reduced skin integrity, long term steroid and immunosuppressive usage, reduced mobility, pain, low haemoglobin/platelets and malnutrition. The Current method of identifying a patient at risk of developing a pressure sore is the Waterlow score. Howerver this is based on an inpatient stay, therefore not relevant to outpatients who will only be in the unit for up to 3 hours during treatment, and sat in the same position for that period of time.

Method: A screening tool, called GOST (Goodgrove Outpatient Screening Tool) was produced, which was adapted from a combination of the Waterlow score and a modified MUST nutrition score. A score of 10 or above indicated that the patient was at a risk of developing a pressure sore, therefore would require a pressure relieving cushion during treatment. A comparison study was undertaken to ensure its reliability and validity. The score the tool indicated was compared against nursing staff intuition and prompts from patients as to whether a patient required a pressure relieving cushion. The comparison study ran for a four week period. Nursing staff gave a pressure relieving cushion to any patient who voiced that their sacrum felt sore or that they were uncomfortable sat for long periods which was recorded. Then the nursing staff used the GOST to ascertain if they scored 10 or above, if they did a cushion was provided, if one had not already been and the results documented.

Results: 65 treatments were included in the study. Of the 65 treatments, 6 treatments $(3.9 \%)$ the nursing staff gave the patient a pressure relieving cushion before completing the GOST. Of those 6 treatments, 4 ( $66 \%$ of those given cushions) scored 10 or above using the GOST. The GOST indicated that 22 treatments of the total 65 treatments $(15.6 \%)$ scored 10 or above and only $4(0.88 \%)$ of those treatments received a pressure relieving cushion prior to using the tool. This meant 18 treatments $(11.7 \%)$ would not have received a pressure relieving cushion had it not been for the tool.

Conclusion: The data collected showed that nursing staff's intuition and patient prompting highlight a small portion of patients that would benefit from a pressure relieving cushion. However the GOST highlighted even more patients which would benefit. Therefore to optimise patient care and prevent the risk of a pressure sore developing the GOST should be used as well as nursing intuition. The patients currently attending the unit have no record of a pressure sore. The tool highlights the patients at a higher risk; therefore by providing a cushion we reduce the risk of a pressure sore developing.

Disclosure of Interest: None declared.

NP027

Establishing an outpatient long intravenous salvage chemotherapy program (LICAP) for lymphoma patients, candidate for HDC auto-SCT: Service Development - First of its kind in Middle East- King Faisal Specialist Hospital and Research Center experience

I. Youssef 1,*, M. S. Rauf ${ }^{2}$, I. Maghfoor ${ }^{2}$, S. Akhtar ${ }^{2}$

${ }^{1}$ Department of Nursing Affairs, ${ }^{2}$ Oncology Center, King Faisal Specialist Hospital and Research Center, Riyadh, Saudi Arabia

Introduction: All salvage chemotherapy programs for lymphoma require 4-6 days of administration. At our center this has been done inpatient. In the interest of cost and efficiency, and to avoid delay of elective admissions, 
lymphoma team initiated a program to shift pre HDC auto-SCT salvage chemotherapy to outpatient treatment area.

Method: The team of Lymphoma Service physicians and clinical nurse coordinator (CNC) drafted this program. A "Patient Centered" multidisciplinary team of physicians, CNC, clinic / inpatient nurses, outpatient treatment area staff, Oncology walk-in clinic, radiology, pharmacy, social services (housing arrangement for out of area patients) and patient educator reviewed the program. Resources needed for transition from inpatient to outpatient LICAP, future needs, manpower and limitations were discussed. Selection criteria for outpatient LICAP included; compliant patients with a caregiver and not requiring inpatient management. Proposed outpatient chemotherapy programs were tested at inpatient setting for 8-9 months, then moved to outpatient chemotherapy administration area; a $61 \mathrm{bed} / \mathrm{chair}$ facility with dedicated pharmacy \& supervising oncologists. Initially, two patients weekly and increased as the system streamlined. CNC coordinated all these arrangements. Program was audited every 4 months for early identification of corrective actions.

Results: Our multidisciplinary team was successfully able to establish a mechanism for this project and shifted LICAP to outpatient setting; first regional outpatient LICAP for auto-SCT patients. No excess complication or related mortality/ morbidity was noticed. Clinical data analyses revealed no difference in clinical outcomes when compared with inpatient program. We documented significant cost saving to the institution (evaluated by finance department). Since January 2012 to October 25, 2015, 97 patients received 203 cycles of outpatient LICAP; calculated an approximate cost saving of $72 \%$ with outpatient as compared to inpatient. In addition, we saved 812 hospitalization days during this time. Although not objectively tested but our patients expressed greater satisfaction with outpatient delivery of LICAP.

Conclusion: Our multidisciplinary team successfully established a LICAP in outpatient setting with safety, significant savings and no loss of efficacy. A focal person (CNC) is an essential component of this program to coordinate all services. Prior to the initiation of program, all stake holders should have open discussion to understand their roles. Periodic audits are essential for timely corrective actions. We plan to carry out an objective quality of life assessment in future.

Disclosure of Interest: None declared.

\section{NP028}

Is Point of Care Testing for a Full Blood Count Needed within the Outpatient Haematology Setting?

J. Garneau ${ }^{1, *}$, O. Brokka ${ }^{7}$

${ }^{1}$ HOPD, IMPERIAL COLLEGE HEALTHCARE NHS TRUST, London, United Kingdom

Introduction: The full blood count is a crucial measurement within the haematology setting with many treatments based on the most recent results. As many of the previous inpatient treatments are moving to the outpatients setting it will require an ability to measure this with accuracy and speed. Within our outpatient department we have a dedicated mini-lab to process this vital test Monday to Friday from 0900 to 1600 and results are often available within 10-15 mins. However the hours we are open for patient care are 0800-2000, 7 days a week. During those times we must use the Hospital's main laboratory for results which can take an hour or longer. As more haematology patients are now neutropenic within the home setting neutropenic sepsis is grave concern for us, and any delay in treatment can lead to poorer patient outcomes. These facts lead us to two main questions and they are: will quicker results contribute to a measurable difference in patient outcomes and are point of care machines accurate enough to base treatment decisions on? Multiple studies over the years have indicated that neutropenic sepsis has better outcomes if treatment is commenced within 1 hour of initial fever (Daniels, 2011). With patients coming from home after onset of symptoms the clock has started prior to arrival on the unit. The ability to extend the hours available to patients who require a $\mathrm{FBC}$ result prior to planned treatment can also be beneficial to the overall running of the unit. Our department is continuing to expand and currently within the haematology outpatient umbrella we have; consultant clinic's, Ambulatory Care including auto transplant, a comprehensive apheresis service (WCD, TPE, RCE, SCH, DLI, PBSCH) transfusion service, triage and assessment clinic, day pain service for sickle cell patients as well as routine haematology day care treatments (chemotherapy, iron etc.) Within haematology many groups need these particular results to initiate urgent treatment, such as ITP with new onset of symptoms. The accuracy to point of care testing is also something that needs to be reviewed prior to launching such an undertaking.

Method: With this in mind we trialled 2 different machines and compared their results with the main haematology lab in the Trust. Accuracy, result report style and ease of use were issues we looked at. We trained 4 nurses on the machines to try and minimize user error and get an accurate picture about the usability of this new tool for the bedside nurse. Both machines were similar in user friendliness, length of time to get results, 1-2 minutes and ease of doing daily controls.

Results: The main lab also ran all of the samples to check for accuracy and after analysis of the data gave us their recommendations.

Conclusion: In conclusion the authors of this paper feel that an accurate, easy to use point of care testing for $\mathrm{FBC}^{\prime} \mathrm{s}$ would be a useful and needed tool.

Disclosure of Interest: None declared.

\section{NP029}

The 'Photopheresis Passport' - The development of a patient held record

L. Robertson ${ }^{1, *}$, M. Foster ${ }^{1}$, T. Maher ${ }^{1}$, C. Rushton ${ }^{1}$, C. Swift ${ }^{1}$, J. Ball', J. Mayo ${ }^{1}$, T. Taylor ${ }^{1}$, N. Matthews ${ }^{1}$, C. Barker ${ }^{\prime}$, A. Alfred ${ }^{\prime}$, P. Taylor ${ }^{7}$

${ }^{1}$ Rotherham Photopheresis Unit, THE ROTHERHAM NHS FOUNDATION TRUST, Rotherham, United Kingdom

Introduction: Patients with Graft-versus-Host Disease often present for Photopheresis (ECP) with complex healthcare needs. The presence of anaemia, thrombocytopenia and immunosuppression in these patients increases the possibility for the incidence of associated complications. The insertion of a central venous catheter in those patients with insufficient peripheral venous access presents further problems, the incidence of infective episodes and blocked catheter lumens potentially resulting in the deferral of treatment. However, following the completion of a central venous access device audit it was found that within our patient records there was insufficient documentation regarding central line insertion, care and maintenance. In addition, communication between the referring trusts and ourselves was suboptimal.

Method: We aimed to develop and implement a 'Patient Passport' encompassing all aspects of ECP treatment, enhancing multidisciplinary communication and encouraging the delivery of holistic patient care. This patient hand held record will compliment existing patient information utilised by the ECP department, aimed to bridge the communication gap and ensure excellence of care.

A review of existing patient passports used within the NHS found wide variations in design and content. We were unable to find any document specifically pertaining to Photopheresis treatment. The requirements and aims of the document were therefore discussed by the Photopheresis team and a draft document was formed incorporating key areas of care and information. Draft copies were then disseminated to a selection of patients and the entire ECP team and feedback was obtained at the monthly governance meeting prior to final approval and printing of the document.

Results: The production of a patient held 'Photopheresis Passport'. The record includes sections for documentation of 
central line insertion together with ongoing care and maintenance of the central venous access device. In addition, patient demographic information, a patient diary, medication lists, treatment schedules and useful contact numbers are included.

Conclusion: The initial aim of the passport was to ensure that line insertion and care was appropriately and reliably documented. This idea was further developed providing a finished product that we feel will be a valuable reference tool for healthcare professionals. The accessible patient held record will provide each patient with an individual guide to their personal ECP schedule, ensuring optimal treatment and patient satisfaction.

Disclosure of Interest: None declared.

\section{NP030}

Outpatient Haemopoietic Stem Cell Transplant

N. Gimberiene ${ }^{1, *}$, L. Birtwistle ${ }^{2}$, E. Tholouli ${ }^{2}$, M. Saif ${ }^{1}, F$. Dignan $^{7}$ ${ }^{1}$ Haematology, Manchester Royal Infirmary, ${ }^{2} 27560$, Manchester, United Kingdom

Introduction: Traditionally Haemopoietic stem cell transplants (HPCT) are performed on an inpatient basis. We have developed a protocol to safely perform ambulatory HPCTs for a group of patients, specially designed to suit our local service set up. Our protocol is based on a prior local audit showing that the majority of patients undergoing autologous HPCT remain well until day +5 . Furthermore, those receiving a non-myeloablative allogeneic HPCT using Seattle conditioning remain well throughout the procedure until engraftment.

Method: - Standard operating procedure (SOP) was developed. -Strict eligibility criteria requires that patients must have a designated caregiver. They must live within $\mathbf{4 5}$ minutes drive from a hospital or stay in a hotel room near the hospital.

- Monitoring checklists were developed for both patient/carer and staff.

-Patients/carers must document their temperature and fluid in/out-put on designated sheets.

- Patients have 24/7 access to the haematology triage team.

-The SOP and practice are continuously monitored, audited and adjustments made accordingly.

- Patients receiving their HPCT in the ambulatory setting are reviewed by a consultant twice weekly in clinic.

- On treatment days patients are reviewed by the transplant fellow or a day unit doctor.

- On rest days Transplant CNS contacts the patient at home and co-ordinates their next treatment as per protocol.

Results: Since January 2015 we have performed 11 ambulatory HPCTs, 5 hybrid autologous HPCT with planned admission on day +5 and 6 allogeneic HPCTs. Four patients receiving autologous HPCT were admitted on day +5 and 1 patient was admitted on day +4 due to early onset nausea. Three patients undergoing a Seattle conditioned allogeneic HPCT experienced complications such as nausea, dehydration and incorrect administration of their medications resulting in hospitalisationof about 2 days. After critical review of practice regular antiemetics (instead of PRN) were introduced and all medications were given in a blister pack which was prepared for 4 weeks. Subsequently 2 further patients remained symptom free and well. At no time point has a patient required emergency admission.

Using a specific questionnaire feed back has been extremely positive as patients were able to remain in the comfort of their own home whilst undergoing life changing treatments.

Conclusion: With the strong MDT support ambulatoryHPCTs have been successfully carried out at our unit. This has been implemented without need of additional resources. Patients and their carers have a positive experience during a time perceived as particularly challenging and stressful. The MDT is currently reviewing other conditioning regimens and planning to expand ambulatory HPCTs to other patient groups. This will reduce the number of patients' inpatient stays under isolation conditions, reduce risk of hospital acquired infections, have positive financial implications and more importantly improve patient experience.

Disclosure of Interest: None declared.

\section{NP031}

A Retrospective Study of Missed Appointments in Extracorporeal Photopheresis Treatment

M. D. Mootien 1,*, J. Schoon ${ }^{2}$

${ }^{1}$ Dermatology Skin Cancer, Guy's Hospital, ${ }^{2}$ Guy's and St Thomas, NHS Foundation Trust, London, United Kingdom

Introduction: The Extracorporeal Photopheresis(ECP) department at Guy's Hospital is the UK's largest. In 2014 extensive work was done to maximise the number of patients treated whilst maintaining staffing numbers ${ }^{[1]}$; and data on the number of missed or cancelled appointments were recorded. This study looks at reasons why appointments were missed with a view to improving efficiency.

Method: A retrospective analysis of data was performed. These data are recorded weekly by nursing staff working in the clinic from cancellations recorded on the handover during the week. Between December 2012 and August 2015477 missed appointments were recorded. Data included: date, hospital number, and reason the appointment was missed. From this a thematic index was devised, categorising why ECP appointments were missed.

Results: Of the 477 missed treatments analysed 65\% $(n=310)$ were due to illness. Eleven other categories were used: $0.8 \%$ $(n=4)$ were missed due to other medications/treatments; $6 \%(n=27)$ did not proceed due to lack of venous access; in $4 \%$ $(n=21)$ the patient refused or cancelled treatment; in 3\% $(n=14)$ treatment was stopped but future appointments had not been cancelled; for $0.4 \%(n=2)$ the patient was unable to get to the appointment due to problems with hospital transport; $0.6 \%(n=3)$ of patients missed appointments due to bereavement/family issues; in $9 \%(n=43)$ no reason was stated; $4 \%(n=19)$ had problems with their blood results; $1 \%$ $(n=6)$ were deceased; $4 \%(n=21)$ of appointments were booked in error and $1 \%(n=5)$ were labelled other.

Conclusion: This research highlights some of the difficulties in maximizing efficiency in the service. In 2014/15 the 61 recorded missed appointments resulted in $£ 83,326$ lost income. This study included unrecorded late cancellations. Almost two-thirds of appointments missed were due to illness or infection, circumstances that are difficult to control, particularly for a patient cohort who are often immunocompromised. 477 recorded missed appointments represents approximately half the total number of appointments missed; as treatment is on two consecutive days, and in most cases data were recorded for only one missed treatment. Therefore the real cost of missed appointments is likely to be much higher.

Changes have been introduced to reduce the number of missed appointments. Clearer guidelines for how to record missed or cancelled appointments have been implemented, as well as a more robust system for notifying patients of appointments. Implementation of a new text messaging system means patients are contacted three to five days prior to their appointments, and nursing staff are required to contact all patients by telephone the day before treatment. Results of these changes will continue to be measured. Providing a high quality and efficient service remains a top priority for the ECP team.

Disclosure of Interest: None declared.

\section{NP032}

Telemedicine project assistance to patients post-hematopoyetic stem cell transplant (HSCT)

M. Salinero ${ }^{1,2, *}$, A. de León ${ }^{1}$, M. García ${ }^{1,2}$, B. Rodríguez ${ }^{1,2}$, L. Vázquez ${ }^{1,2}$, M. D. Caballero ${ }^{1,2}$

${ }^{1}$ Hematología, COMPLEJO HOSPITALARIO UNIVERSITARIO DE SALAMANCA, ${ }^{2}$ Intituto de Investigación Biomédica (IBSAL), Salamanca, Spain 
Introduction: The increase number of transplants and the better survival of patients require the implementation of new strategies to reduce the workload in the transplantation center and, to avoid patients travel to the transplant center.

Telemdicine is a new area of health where medical information is transferred through audiovisual media, for the purpose of remote doctors and nurses visits or examinations. In this new scenario Telemedicine and Telenursing means the possibility of globalize the HSCT control process using communication networks and decreases number of Transplant medical center visits.

The purpose of this project is to substitute "face to face" visit in candidate patients.

Method: Inclusion criteria:

Patients in early stage (less than 100days) post- transplantation without complications

Patients of more than 100 days post- transplant with no evidence of active GvHD.

Basically those that only require regular monitoring and analytical tests to verify that the evolution of the process is appropriate: long term survivors, patients who do not require immunosuppressants and/or stable patients with GvHD, as well as immunosuppressive patients, even in the period of post-transplant day -100 and which require more than a weekly check.

All units of Hematology in Castilla and Leon have the computer application "Jimena", which provides help to the professionals in the health care act, and also in the registration of the assistance.

For the start-up of this program the conformity of medical center sanitary personal is required. The Transplant medical center allocates a contact nurse expert in transplant. She/he will be supported daily by a hematologist of the transplant team.

During the virtual visit, the nurse will evaluate the physical symptoms, vital signs, medical history, and any side effects from the allo-HSCT. At the end of the visit, the nurse and the doctor in the Transplant Unit will check on site all the blood tests and will review medical orders and general recommendations as well as they will program the next visit. The patient will evaluate the event using a survey to assess the acceptance of the virtual visit. The surveys will be written and should take around 15 minutes to complete.

Results: They are based on assessing the main objectives of the project analyzing:

1. Effectiveness of telematics assistance in monitoring patients receiving $\mathrm{HSCT}$.

2. Reduction the number of visits to the transplantation center. 3. Satisfaction of: patient, caregiver, centers of origin and transplant center.

4. Economic impact.

Conclusion: The development of virtual visit will improve the quality of life of patients and will decrease the work load of the transplant unit without affecting quality of care.

Disclosure of Interest: None declared.

\section{NP033}

Management of the autologous stem cell transplant in ambulatory care: home care or day care unit?

C. Gallego ${ }^{1, *}$, A. Hernando ${ }^{1}$, S. Segura ${ }^{1}$, N. Arab ${ }^{1}$, M. Carreño ${ }^{1}$, J. Guell', A. Gaya', G. Gutierrez', M. Valverde', F. Fernandez ${ }^{1}$

${ }^{1}$ Hematology and Oncology, Hospital Clinic, Barcelona, Spain

Introduction: Management of autologous stem cell transplant (ASCT) patients in ambulatory care is a real and safe alternative to the conventional model. In the literature, different strategies are presented for the management of them, but a considerable number of these are performed only in Day Care Units (DCU), frequently without a specific team, frequent visits in the DCU and high readmission rates. In our institution we have 15 years of experience with a monographic Home Care Unit (HCU) based in a mixed model, where the major part of assistance is performed at home by expertise nurses and only needs to come to Hospital in case of clinical complication or transfusion. Our HCU model represents a next steep to offer a personalized assistance, with a system adapted to the necessities of the patients into the procedure.

Objective: To demonstrate the benefits of "homecare model" in the ambulatory management of ASCT patients.

Method: Revision of the literature to introduce differences and key points between the two models of attention1. HCU have a reference team (physician-nurses) who treat the patient; 2. Infections: minimizing nosocomial infection risk; 3. Empowerment: personalized attention (medical and nursing) with an adapted program to the real necessities of the patients and caregiver; 4. Safety: homecare model include a "Support Unit" available $24 \mathrm{~h}$ a day to attend emergencies and complications. Results: Fever incidence was present in 153 of 247 (62\%) patients, with a documented infection in 24 cases of the 153 that had fever (16\%) and only one case with a multi-resistant Pseudomonas aeruginosa. Only 26 patients need a readmission $(10.5 \%)$, the majority by fever. The median (range) days treated by HCU was 14 (2-27) with 11 (4-26) visits at home per ASCT case. Thirty per cent $(74 / 247)$ of the patients needs to stay $24 \mathrm{~h}$ in observation on HCU support unit and 22\% (54/247) needs two visits per day by the nurse at home.

Conclusion: The option to treat ASCT in a DCU represents and easy form to treat these patients for the institutions because they have the circuit and don't need to implement a new device. In our experience, a monographic HCU permits to optimize the clinical results of the ambulatory ASCT programs adding into the clinical protocol safety criteria, personalized attention and empowerment in the process both the patient and caregiver.

Disclosure of Interest: None declared.

\section{NP034}

Welcome Programme for new nurses in a Hematology Unit (HU) and Hematopoyetic Stem Cell Transplant Unit (HSCTU)

M. C. Gonzalez Rodriguez ${ }^{1, *}$, H. Garcia Alonso ${ }^{1}$, S. Martin Martin ${ }^{1}$, M. D. Ferrero Calderon ${ }^{1}, M$. I. Iñigo Elena ${ }^{1}, M$. B. Rodriguez Martin $^{1}$

${ }^{1}$ Servicio de Hematologia y Transplante de medula osea, Hospital Clinico Universitario de Salamanca, Salamanca, Spain

Introduction: $\mathrm{HU}$ and HSCTU from the University Hospital of Salamanca (UHS) are special units that require very specific health care as well as qualified and very well trained nurses, to provide the best care for these patients suffering from very severe diseases who are admitted to receive so complex procedures as is HSCT. One of the most important issues is to avoid any mistake in the preparation, manipulation and administration of treatments; if they appear, inmediate resolution of adverse events is mandatory; their consequences derive in health repercussions with impact on economic and, in particular with human costs, generating distrust with the professional and with the healthcare system. With the arrival of new staff at the Unit, it was clear that there was a need to make a welcome programme, with established, explicit and detailed protocols and procedures on how the unit works, the way to prepare and administer medication and the specialization of the nursing care for these specific patients. All of these measures were approached with the objective of the patients' safety and comfort, in order to improve hematologic care, in addition of ensuring a safe medical practice and reducing daily mistakes.

Method: First, we held a training course called "Hematologic Nursing Care". The teachers were four qualified nurses and the head nurse from HU and HSCTU who were highly trained and had experience in the clinical field. The main topics were: Types of transplants and their care (Autologous/Allogeneic), Mains treatments, Types of chemotherapy, Types of insulation, Emergencies and fever management at UH/HSCTU. Unit protocols were updated and available for the new staff. We also developed a poster including the most used medications 
in the unit, how to prepar them, the proper doses and their compatibilities. This poster is actually in the nursing room and in the personal nurse folder. As an essential part of this work "A practical guide on how to prepar and administer intravenous medicines" from UHS was employed, made from technical files from each type of medication. We also used the "Handbook on injectable drugs" Trissel LA. Finally, we carried out a Management and Security session, which was conducted by the Prevention of Risk at Work Management team at HUS. Results: We succeeded in training and providing information for all the new staff of nurses at HU and HSCTU and updated information, procedures and protocols regarding nursing techniques and the preparation and administration of medication. Therefore, improving healthcare quality and identifying and reducing risks.

Conclusion: A Welcome Programme is mandatory to get a trained and qualified multidisciplinary team, necessary for ensuring a high level of quality care, through safe practices, reducing risks to the lowest possible level in these complex medical Units.

Disclosure of Interest: None declared.

NP035

Comparative analysis of different CVC dressing types convenience and economic effectiveness in allogeneic hemopoietic stem cell transplant recipients

O. Ivanova ${ }^{1, *}$, J. Simakina ${ }^{1}$, M. Ermolova ${ }^{1}$, J. Oparina ${ }^{1}$, E. Goncharova ${ }^{1}$, L. Zubarovskaya ${ }^{1}$, B. Afanasyev

${ }^{1}$ First Pavlov State Medical University of St. Petersburg, Saint-Petersburg, Russian Federation

Introduction: The care for critically ill patients in our clinic requires in most cases the central venous access for therapeutic interventions and diagnostics. The use of central venous catheters (CVCs) has its drawbacks, one of which is development of catheter-associated infections (CAI) found in $20-55 \%$ of patients with sepsis. CAl development leads to significant raise of treatment costs. The prevalence of CAI depends on intraluminal and exit site infections prophylaxis by using appropriate CVC maintenance equipment and techniques.

Method: A total of 112 of allo-SCT recipients with AML, ALL, $C M L$ and $A A$ were divided into 3 clinical groups based on dressing type used. All patients had CV accesss via v. jugularis interna dextra. In the $1^{\text {st }}$ group $(n=38)$ sterile self-adhesive non-woven pads (Cosmopor) were used. In the $2^{\text {nd }}$ group $(n=37)$ transparent Tegaderm Film dressings were used. In the $3^{\text {rd }}$ group $(n=37)$ transparent gel pads Tegaderm CHG were used. Nurses from CVC care team evaluated patients' complaints and performed catheter site monitoring for possible presence of local inflammatory changes, allergic reactions and CVC displacement. In addition, dressings changing convenience, catheter site cultures results and economic aspects were analyzed.

Results: The catheter exit site bacterial colonization was lower in patients with Tegaderm CHG dressings (3.4\%), than in patients with non-woven pads $(5.7 \%)$ or Tegaderm Film $(5.3 \%)$ dressings. All $1^{\text {st }}$ group (non-woven pads) patients reported frequent dressing deformation requiring its replacement and skin discomfort due to residual adhesive material. Patients from the $2^{\text {nd }}$ (film pads) group complained of skin contraction in movement (23\%) dressing deformation (22\%), itching (18\%), while $37 \%$ of patients had no complaints. In the $3^{\text {rd }}$ group (transparent gel pads) $92 \%$ of patients were satisfied with dressing performance, while $2 \%$ reported itching and $6 \%$ movement restriction. From nurses' points of view non-woven pads were inconvenient due to the difficulties of exit site monitoring, need of frequent changing of soiled, wet or deformed dressings. Transparent films were more convenient due to better visual control and less dense change schedule (once 2-3 days). Gel pads were most convenient as they required changing only one in 5-7 days and provided good catheter fixation. The daily cost was greater for daily changed non-woven pads (4.35 euro), than for film pads (2.67 euro) or gel pads ( 1.72 euro).

Conclusion: Transparent gel pads (Tegaderm CHG) is optimal in post-transplant patients due to less frequent change, which leads to less frequent skin traumas and exit sites infections, is more convenient for patients and nurses. In addition, although gel pads are relatively more expensive, their use provide economic advantages to lower daily cost (1.5-2.5 less than other dressing types) and less frequent CAls.

Disclosure of Interest: None declared.

\section{NP036}

Help, Mum or Dad has cancer: literature search on how nurses can support children and parents

I. Agreiter ${ }^{1, *}$, R. Procaccia ${ }^{2}$, D. Cevales ${ }^{3}$

${ }^{1}$ Division of Haematology and BMT Centre, Regional Hospital, Bolzano, ${ }^{2}$ University Ecampus, Novedrate, ${ }^{3}$ Psychological Service, Regional Hospital, Bolzano, Italy

Introduction: According to estimates of the National Cancer Institute, $24 \%$ of adults with cancer are parenting children under 18 years. Many studies report how to support parents when children have cancer or children with cancer, but less has been published how to support children or help parents coping whit the diagnosis of cancer.

Method: Entering the key words "children experience "parental cancer"'" and "children "parental cancer" intervention"'" in PubMed, a total of 38 articles were selected. Twenty-five lived up to the author's expectations and were analysed.

Results: In case of parents' cancer, the age of children doesn't play a fundamental role, because already in the early childhood, children understand that there is something wrong in the family: more phone calls, more relatives' visits, parents get gifts or flowers and nobody tells them what is happening. Nurses can be a prompt support from the onset of the diagnosis of cancer, advising parents to tell the truth as soon as possible to their children. Later, nurses could involve the children in department tours where they can see and have a real picture of the location where their parents get chemotherapy in the day hospital. Some centres have strict visitor norms, it is important for children to see the hospitalization room, and also for parents to see their children, therefore are nurses the unique figure to act as intermediate and make physicians understand the importance of children's visit in hospital.

Frequently adolescent children, especially if mothers get cancer, have to do housework, to look after younger siblings, to go to school, do homework and the time for friends or hobbies is lacking. It is important that children or adolescents can remain as such and do the same activities as before the parents' disease. Even in this case, nurses can inform parents how to avoid children's renunciations. Adolescents wish to get detailed information about the parents' disease from physicians, but often they use technical terms, which are not understandable and need help from nurses.

Conclusion: During hospitalization, most of the patients have enough time to think about a lot of topics. Children are for parents a huge theme, and if they get support from nurses how to manage easier the situation at home being in hospital, the road to recovery will be faster.

Disclosure of Interest: None declared.

\section{NP037}

Support and information group for patients and their families in bone marrow transplant unit

J. Basin ${ }^{1, *}$, L. Katzir ${ }^{1}$

${ }^{1}$ Bone Marrow Transplant Unit, Rambam Health Care Campus, Haifa, Haifa, Israel

Introduction: During a bone marrow transplant some patients develop severe complications such as GVHD, respiratory 
complications (pulmonary bleeding or severe pneumonia), etc. The treatment for this type of patients is prolonged and intensive; it tends to focus on the medical aspects, less so on the psychological impact. Some of these patients become terminally ill. This takes an emotional toll on the patients and their families who are unable to come to terms with death and cope with grief due to lack of involvement of a multidisciplinary team. To address this need we developed information and support group with two primary goals: 1. Provide psychological and emotional support to match every stage of the medical treatment for the patient and their loved ones. 2. Create professional communication circle around the patient.

Method: To develop a support system for this type of patients we have created a working group that includes physician in charge, a social worker and a nurse. This team meets weekly to review both immediate and long-term goals, to plan specific therapy for individual patients and their families based on their treatment needs. Later meetings with patients and their families take place, each session is conducted according to the needs of that patient. The frequency of meetings is also adjusted per patient and can take place one to four times a week. There is generally no need for the participation of all members of the group, but this depends on the needs of the patient. At the end of each week all nursing and medical staff gets an update for the week.

Results: This support group will contribute to a more normal communication and improve the transfer of information between the teams. In addition, each patient receives close multidisciplinary support. This support helps a patient and family cope daily with the disease, particularly in the terminal stages of the illness. After a few months of operation of the support group, we plan to develop tools for evaluating the group's efficiency.

Conclusion: Nursing care is a heterogeneous concept of treatment that consists of assessment, planning, implementation, coordinating, monitoring, and evaluating the of options and services required to meet the client's health and service needs. A nurse plays an important role in identifying patients with special needs and operates and coordinates the work triangle "doctor-nurse-social worker." Despite the fact that the support group is only beginning to act, we hope to expand the activities and positive contribution to the work of medical staff and help patients and families cope with the disease.

Disclosure of Interest: None declared.

\section{NP038}

Hemato- oncology nurses, treating compassion fatigue in hemato-oncology department through group therapy M. Furer ${ }^{1, *}$, V. Latergaous ${ }^{1}$, L. Katzir ${ }^{1}$, Z. Rapaport ${ }^{1}$, E. Muller ${ }^{1}$ ${ }^{1}$ rambam health campus, haifa, Israel

Introduction: Compassion fatigue has been defined as a combination of physical, emotional, and spiritual depletion associated with caring for patients in significant emotional pain and physical distress. Compassion fatigue is identified as a unique form of burnout that affects caregivers. Most caregivers, especially in oncology, are intent to help others by providing empathetic care for patients with critical physical, mental, emotional, and spiritual needs. These caregivers often become victims of the continuing stress, as a result of their exposure to extreme illness and overwhelming suffering.

Method: As a professional oncology nurses, who are qualified and trained facilitators of psychodynamic group therapy, we led dynamic support groups for the hemato-oncology staff. The type of therapy we focused on - was intensive short term psychodynamic sessions by two co- therapists working together. The goals were to create an emotional and safe space for all the participants, to encourage them to deal more effectively with their own emotions, thereby prevent the potential of secondary traumatization. The group contained 12 nursing and medical oncology staff, which came from diverse background (beginner / professional nurse, ordinary nurse/head nurse), at different stages of the treatment process. The group setting was safe and supportive, group met once each week for an hour and half, 7 therapy sessions. All the sessions were transcribed and processed in order to define the main theme for the following sessions.

Results: At the end of the process we evaluated the effectiveness of the intervention by qualitative interview of all the participants. The participants shared emmotional difficulties from their clinical experience and more over expressed the added value of getting a safe space to cope with issues such a stress in the workplace, loss, anxiety, depression, grieving, fear of death. These issues came out from personnel interventions we made.

Conclusion: Compassion fatigue affects job satisfaction, emotional and physical health, decrease the quality of care and increase staff turnover. Psychodynamic supportive group therapy by the qualified oncology nurses, who are qualified and trained facilitators of psychodynamic group, could be a significant cost effective method for every aware health organization in order to prevent this phenomenon. It is beneficial for both the staff and the patients to facilitate intervention strategies for the staff, with the purpose of developing self care for the staff and increasing the quality of care for patients.

Disclosure of Interest: None declared.

\section{NP039}

Relationship between distress and prolonged hospitalization after allogeneic hematopoietic stem cell transplantation

N. Yokota ${ }^{1, *}$, T. Kamimura ${ }^{2}$, Y. Ito ${ }^{2,3}$, T. Aoki ${ }^{2}$, H. Muraoka ${ }^{1}$, S. Miyazaki ${ }^{\prime}$, M. Yanagisako ${ }^{1}$, T. Miyamoto ${ }^{4}$

${ }^{1}$ Department of Nursing, ${ }^{2}$ Department of Hematology, Harasanshin Hospital, Fukuoka, ${ }^{3}$ Department of Internal Medicine, Kyushu University Beppu Hospital, Beppu, ${ }^{4}$ Medicine and Biosystemic Science, Kyushu University Graduate School of Medical Sciences, Fukuoka, Japan

Introduction: Detailed information about relationship between distress and prolonged hospital stay in patients who underwent allo-HSCT has not been sufficiently documented.

Method: Distress in patients who underwent allo-HSCT was prospectively evaluated by Hospital Anxiety and Depression Scale (HADS) before and every 30 days after transplantation. We divided the patients into two groups: "the discharge group" including patients who were scheduled for discharge after transplantation and "the prolonged hospitalization group" including patients who were expecting a prolonged hospital stay of 90 days after transplantation. The primary endpoint was a difference in the level of HADS total score before and 90 days after allo-HSCT between the two groups. Sample size $(n=32)$ was calculated based on a pilot study. The institutional review board of Harasanshin Hospital approved this study.

Results: Out of the 32 patients enrolled, 11 discontinued HADS analysis, of whom two suffered from poor physical conditions and nine died. The remaining 21 were divided into the discharge $(n=10)$ and prolonged hospitalization groups $(n=11)$. Changes in HADS scores are shown in Figure 1. Changes from pre-transplant to day 90 in HADS total, depression, and anxiety scores in patients between the discharge and prolonged hospitalization groups were as follows: (a) HADS total score, $-1.5 \pm 2.4$ vs. $3.6 \pm 5.2$ $(P<0.05)$; (b) anxiety score, $-1.3 \pm 1.6$ vs. $0.18 \pm 3.0(P>0.05)$; and (c) depression score, $-0.2 \pm 2.7$ vs. $3.5 \pm 3.6(P<0.05)$ (paired t-test). Figure 2 shows serial changes in HADS scores. In the prolonged hospitalization group, HADS total score was significantly higher 90 days after transplantation than before transplantation.

Conclusion: Our results suggest that a prolonged hospital stay can increase transplant patients' distress. Therefore, we should pay more attention to the observation and management of 


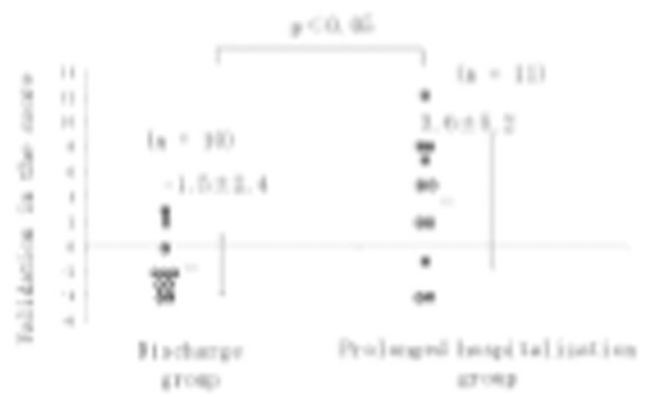

th Osup in the leswly foe assety ware

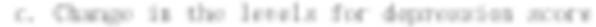

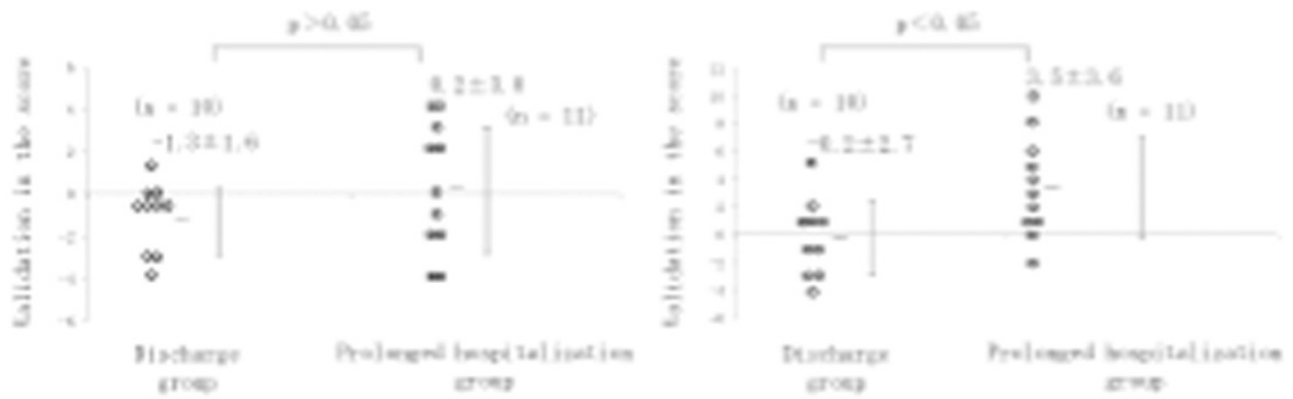

Figure 1. Changes in the levels of HADS scores

Dimcharne strup

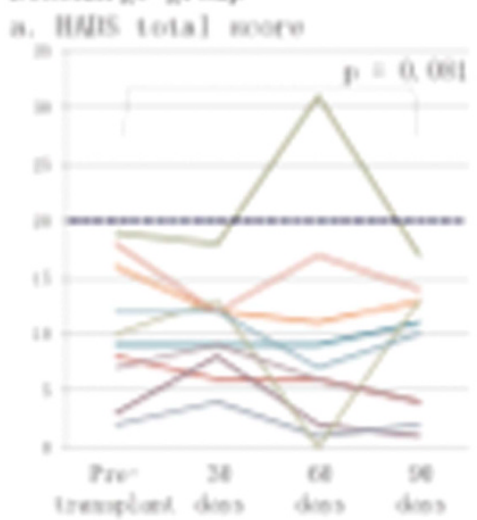

Prolonged bogitalizatioe sroup 4. HNis telal soore

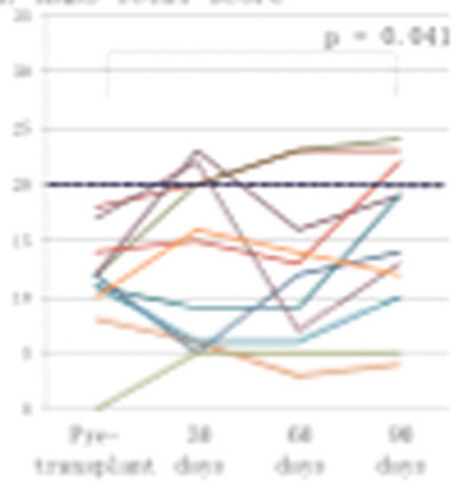

b. Hus arxiely stare
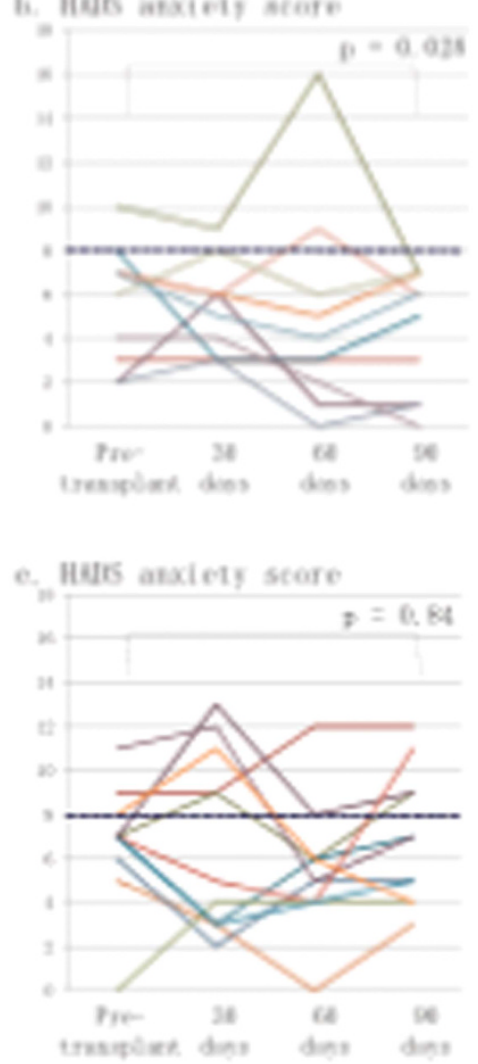
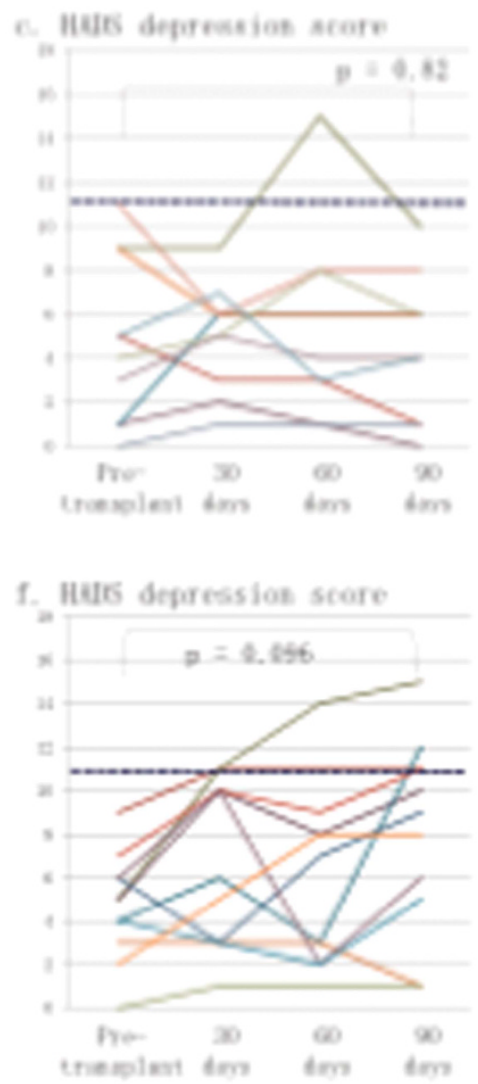

Figure 2. Serial changes in HADS scores 
psychological health status of patients with a prolonged length of hospital stay even if they do not look like gloomy. Disclosure of Interest: None declared.

\section{NP040}

Spiritual care for stem cell transplant patients: A nursing perspective

S. Ghazi $i^{1, *}$

${ }^{1}$ King Abdulaziz Medical City, Riyadh, Saudi Arabia

Introduction: Patients undergoing stem cell transplantation require every facet of care to be considered. The care related to the physical, social and psychological aspects are seemingly managed well and seen within the scope of practice for registered nurse, however spiritual care is considered to have less attention by nurses. Numerous studies have emphasized the importance of spiritual care and highlighted many challenges nurses are encountering in their process of providing spiritual care. The aim of this study was to identify the concerns from nursing perspectives in the area of spiritual nursing care for oncology patients and more particularly for stem cell transplant patients. The core uniqueness of the context of this research was the multicultural participants that represent the multinational nursing staff of the research setting in an internationally accredited quaternary care hospital in Saudi Arabia.

Method: A qualitative research approach of interpretive description process of inquiry was utilized. Audio-taped semi-structured interviews were used for data collection from 12 oncology nurses that were voluntarily participated and purposefully selected to explore their lived experience.

Results: Five themes in addition to several subthemes derived from this research that was recognized as factors influencing spiritual nursing care of oncology patients. The main themes are (a) cultural diversity, (b) nurses' readiness, (c) administrative support, (d) part of nursing care, and (e) patient and family needs. The resultant themes and subthemes revealed that nurses are facing many challenges and opportunities at different levels in their provision of spiritual care and more effort is needed to provide culturally and spiritually congruent care. Moreover, the findings confirmed that nurses have good opportunity to meet patients' spiritual needs. To support these themes the words of the participants will be used.

Conclusion: This research is the first of its kind in the study of spiritual nursing care as explored and described by a multifaith group of nurses caring for a monotheistic group of oncology Saudi patients. The study findings have implications to clinical nursing practice, administration, education, as well as future research. The finding revealed that there are different areas for improvement in providing spiritual nursing care. These improvement areas could be achieved by collaborative effort between bedside nurses, nursing education, administration, and continuing to conduct more researches.

Disclosure of Interest: None declared.

\section{NP041}

The lived experiences of patients hospitalised in protective isolation: a qualitative inquiry

V. Biagioli, ${ }^{1,}$, M. Piredda ${ }^{2}$, R. Alvaro ${ }^{1}$, M. G. De Marinis ${ }^{2}$ ${ }^{1}$ Biomedicine and Prevention, Tor Vergata University, ${ }^{2}$ Research Unit in Nursing Science, Campus Bio-Medico di Roma University, Rome, Italy

Introduction: Protective isolation is considered a necessary precaution for preventing infection in patients with haematological malignancies undergoing haematopoietic stem cell transplantation (HSCT) in many centres worldwide. Although protective isolation aims to benefit patients' health, it could result in a worsen quality of life, since HSCT patients may suffer because of the restriction of contact with the outside world and with loved ones. In order to support an evidence-based statement about psychological implications of protective isolation, patients' perceptions of being isolated need to be examined further. Therefore, the aim of this study was to explore the lived experience of patients with haematological malignancies who had been hospitalised in protective isolation for autologous HSCT.

Method: A descriptive phenomenological study was conducted in a University Hospital in Italy with 9 patients who had undergone autologous HSCT (mean age $=57$ years). During summer 2015, two researchers interviewed patients awaiting for their weekly visit that is performed until 100 days posttransplant, and asked about their stay in isolation. An unstructured interview technique was adopted for data collection in order to allow maximum freedom of expression for the informants. All the interviews were digitally audio-recorded and lasted between 22 and 55 min (mean duration $=35 \mathrm{~min}$ ). Giorgi method of analysis was used to describe the essential structure of the lived experience in protective isolation from the patients' perspectives.

Results: Nine themes emerged from the data analysis: isolation is a defence, threats which patients have to defend from, the burden of the defence, external strategies for defence, inner strengths for defence, rules for defence, defending loved ones, outcomes of the defence, the defence continues. The essential meaning of protective isolation was the possibility to defend oneself from threats such as infections. This defence was not a siege for the patient, but an active battle which had to be conducted according to the rules of the hospital setting. To help defend themselves from the burden of the experience, patients believed to have many strategies, both internal such as a positive attitude, and external such as technology. Patients wanted to fight also for defending their loved ones. If the defence had success, patients may have achieved positive outcomes, with the growth both of their inner power and their blood cells. Their defence did not end at the discharge, but continued also at home and for many days after their transplant.

Conclusion: The perception of protective isolation as a defence is central to the experience of patients, along with an enhanced need for defending both themselves and loved ones. Since patients need to feel active fighters against cancer, and try to find effective strategies for their defence, it is vital to provide emotional and social support.

Disclosure of Interest: None declared.

\section{NP042}

Fail to Engraft Post Allogeneic Stem Cell Transplantation-Stress on Nursing Care Y.-C. Chuang ${ }^{1, *}$, Y.-C. Huang ${ }^{1}$, F.-M. Tien ${ }^{2}$, C.-F. Chang ${ }^{1}$, C.-T. Lin ${ }^{1}$ ${ }^{1}$ Tai-Cheng Stem Cell Therapy Center, ${ }^{2}$ National Taiwan University Hospital and College of Medicine, National Taiwan University, Taipei, Taiwan, Province of China

Introduction: A 43-year-old female patient with myelodysplastic syndrome underwent $7 / 8$ partial mismatched unrelated allogeneic peripheral blood stem cell transplantation (PBSCT) with reduced intensity conditioning. She experienced secondary graft failure after transient leukocyte recovery. She received second allogeneic PBSCT from the same donor, and primary graft failure was diagnosed.

The transplantation team decided to carry out third transplantation from her sister-using a haploidentical protocol with bone marrow stem cells (BMSC) and peripheral blood stem cells (PBSC). This patient expressed high self-pressure and hopelessness, not only for the potential risk of repeated graft failure but also a sense of the sacrifice of her sister-risk of donating BMSC and PBSC at the same time. She realized imminent mortality if the transplantation failed again. Beyond taking care of physical needs, nursing staffs should also emphasize on alleviating her enormous mental stress.

Method: 1. Assign experienced caregivers at bedside, and take care of physical and mental needs simultaneously. Lighten the burden of her family. 
2. Disclose further treatment plans and potential side effects, and guarantee what nursing staffs would help.

3. Arrange face-to-face communication with other patient who experiences graft failure and salvaged successfully. They can encourage her, and share the thoughts.

4. Provide proper donor care and ensure donor's safety and comfort. Ask the donor and her family to kindly encourage her. 5. Contact social workers and other medical resources to provide possible economic support.

6. Religionary support.

7. Family meeting and re-build their confidence on the transplant team.

Results: After meeting with the previous successfully salvaged patient who also experienced graft failure, she became more optimistic and accepted the therapies. She turned out to be active and open about expressing her sufferings. It was worth mentioning that the patient had no good alternative treatment options but to choose from either receiving the third transplantation or confronting her death due to prolonged neutropenia. On the spiritual aspect, the religionary support was indeed important and more beneficial to the patient.

We held family meetings which provides the chances of listening and discussion prior to major medical decision makings, and ensured that transplantation team's goal was in line with their anticipation, and was from misunderstandings. Help from social workers also warmed up their heart.

Conclusion: Through these ways, we could provide a comprehensive care which not only relieve her negative emotions but also motivate her to express what she deeply cared about. Besides satisfying patient's physical needs, it cannot be emphasized more that caring her spiritual aspect gave invaluable support for this patient through the entire treatment course.

Disclosure of Interest: None declared.

\section{NP043}

Impact of autologous hematopoietic stem cell transplantation on the quality of life of systemic sclerosis patients

É. A. D. O. Cardoso, ,*, A. L. Guimarães ${ }^{1}$, J. Garcia ${ }^{1}$, J. Dias ${ }^{1}$, D. Moraes ${ }^{1}$, K. Pereira ${ }^{1}$, A. B. Stracieri ${ }^{1}$, B. Simões ${ }^{1}$, M. C. Oliveira $^{\prime}$, M. A. Santos

${ }^{1}$ USP, Ribeirão Preto, Brazil

Introduction: Autologous hematopoietic stem cell transplantation (AHSCT) has been investigated as a promising therapeutic alternative for systemic sclerosis (SSc) patients. Because it is an innovative therapy for SSc, there is urgent need for studies that assess not only efficacy of the procedure, but also how it affects patient lives.

Objective: To evaluate the impact of AHSCT on the Quality of Life (QoL) of SSc patients, before and two years after the procedure.

Method: This is a descriptive and exploratory study, with longitudinal cross-section of a cohort of patients treated at a University Hospital in the state of São Paulo, Brazil. The MOS SF-36 scale consists of 36 items that assess two main components: the physical health component (PHC) and the mental health component (MHC). The PHC encompasses the following domains: physical functioning (PF), role limitations due to physical problems (RF), bodily pain (BP) and general health perceptions (GH). The MHC comprises: vitality (VT), social functioning (SF), role limitations due to emotional role functioning (RE) and general mental health (MH). Results for each domain range from 0 to 100 , zero being the worst and 100 the best state of health. Data were collected at admission, and 2 years after AHSCT. Instruments were applied individually. Results were subjected to statistical analyses. Significance levels were established at $P<0.05$.

Results: Twenty-two SSc patients, 14 (64\%) females, with mean(SD) age of 39.8 (9.64) years were included and evaluated before and 2-years after AHSCT. Our results indicate improvement of QoL in SSc patients post-transplantation. After AHSCT, we observed an increase in PF, RF, BP, VT, SF, $\mathrm{MH}(P=0.01)$ and $\mathrm{GH}(P=0.05)$, while RE remained stable (Table).

Table - Distribution of SF-36 scores, before and 2-years after AHSCT

\begin{tabular}{|c|c|c|c|c|c|c|c|}
\hline \multirow[t]{2}{*}{ Domain } & \multicolumn{3}{|c|}{ Pre-HSCT } & \multicolumn{3}{|c|}{ Post-HSCT1 } & \multirow[t]{2}{*}{$P$-value } \\
\hline & Mean & $S D$ & Median & Mean & $S D$ & Median & \\
\hline PF & 41.3 & 33.9 & 35 & 67.2 & 28.4 & 85 & 0.01 \\
\hline RF & 23.8 & 40.4 & 0 & 42.3 & 30.0 & 50 & 0.01 \\
\hline $\mathrm{BP}$ & 46.8 & 32.9 & 46.5 & 71.1 & 25.9 & 67.3 & 0.01 \\
\hline $\mathrm{GH}$ & 52.2 & 27.0 & 55 & 66.6 & 14.3 & 67 & 0.05 \\
\hline VT & 55.2 & 30.1 & 60 & 67.2 & 20.3 & 75 & 0.01 \\
\hline SF & 20.3 & 23.2 & 62.5 & 68.5 & 27.6 & 68.7 & 0.01 \\
\hline $\mathrm{RE}$ & 54.5 & 45.4 & 50 & 54.5 & 41.8 & 33.8 & - \\
\hline $\mathrm{MH}$ & 59.4 & 24.5 & 64 & 64.6 & 23.8 & 68 & 0.01 \\
\hline
\end{tabular}

Conclusion: The increase in QoL at late post-transplantation periods may indicate that patients do not face the constant possibility of disease reactivation, translated by clinical stabilization or improvement after the procedure. These results may be interpreted as positive outcomes of AHSCT for SSc.

Disclosure of Interest: None declared.

\section{NP044}

Quality of life in patients with multiple myeloma

K. Jo ${ }^{1, *}$ J. Ban ${ }^{2}$, J. Yoon ${ }^{2}$, S. Kook ${ }^{2}$, H. Yoon ${ }^{2}$

${ }^{1}$ Seoul ST Mary's hospital, '2Seoul ST Mary's hospital, Seoul, Korea, Republic Of

Introduction: Quality of life has become an important outcome in hematologic malignancy. Multiple myeloma is the second most common hematologic malignancy. Recently, treatment in multiple myeloma has advanced and the survival of patients with disease has significantly improved. So, quality of life is an essential part of the management of myeloma patients. The purpose of this study was to identify quality of life in patients with multiple myeloma and the difference of quality of life by their characterisitics.

Method: In this descriptive study, the participants were 189 multiple myeloma patients who had treated a $C$ university hospital in Seoul from July 2014 to December 2014. Data were collected through self-reported questionnaire. Quality of life was evaluated using the European Organization for Research and Treatment of Cancer(EORTC) QLQ-C30 and a specifically designed instrument for use among patients with multiple myeloma known as the EORTC QLQ-MY20. The collected data were analyzed with T-test, ANOVA, Kruskal-Wallis test, Duncan test and Mann-Whitney test.

Results: The mean score for each subscale of EORTC QLQ-C30 was 53.35 for global health status, 73.37 for functional scale, and 31.29 for symptom scale. The mean score for each subscale of EORTC QLQ-MY20 was 60.49 for future perspective, 59.78 for body image, 20.25 for disease symptom and 24.99 for side effect of treatment. The Quality of life were significantly lower in patients who are: female, not employed, depending on sibling for treatement pay, having anemia, having treatment, having high ECOG performance score, and suffering from high grade of peripheral neuropathy.

Conclusion: This survey would help identify characteristics which impact quality of life of multiple myeloma patients. According that, it is necessary to develop appropriate educational strategies and nursing intervention to enhance their quality of life.

Disclosure of Interest: None declared. 


\section{NP045}

Impact of chronic GvHD in the quality of life of patients undergoing allo-hematopoietic stem cell transplant (HCST): Experience at the University Hospital of Salamanca M. Salinero ${ }^{1,2, *}$, M. García ${ }^{1,2}$, A. de León ${ }^{1}$, B. Rodriguez ${ }^{1,2}$, L. López ${ }^{1,2}$, E. Pérez ${ }^{1,2}$

${ }^{1}$ Hematología, COMPLEJO HOSPITALARIO UNIVERSITARIO DE SALAMANCA, ${ }^{2}$ Intituto de Investigación Biomédica (IBSAL), Salamanca, Spain

Introduction: Chronic graft-versus-host disease (cGvHD) is a major cause of late morbidity and mortality after allo-HSCT. Signs and symptoms of cGvHD resemble those of chronic autoimmune diseases, and generally afect more than one organ, especially the skin and oral, ocular and vaginal mucosae.

Several studies show that the development of cGvHD impacts negatively on the quality of life $(\mathrm{QL})$ of the allotransplanted patients. Our study analyzes this impact on our patients.

Method: We offered this study to all our allotransplanted patients and 137 of them, between 12 and 192 months post-allo HCST, signed the informed consent. We used the FACT-EBMT Quality of Life Self-Assessment Questionnaire, version 3. It consists of 54 questions covering their physical, familial, social, emotional and functional wellbeing by scoring them from 0 (never/nothing) to 4 (much/frequently). In addition, patients can also scored their overall situation in each of these areas from 0 to 10 . Statistical analyses were performed using the SPSS software, version 20.

Results: In our series of patients, donor was a sibling HLA-identical in $64.2 \%$ of cases and unrelated in the remaining $34,8 \%$. $67,9 \%$ received a non-myeloablative conditioning. Of the 137 patients included in the study, $35.8 \%$ of them had active cGvHD requiring systemic immunosuppression at the time of filling the questionnaires.

Patients with active cGvHD valuated more negatively the questions about physical wellbeing, especially those related to eye discomfort $(53.1 \%)$, mouth $(35.4 \%)$, and pain $(22.4 \%)$ fatigue $(29.8 \%)$, energy loss $(30.6 \%)$, increased need for rest (51\%), physically limited (22.4\%), and those associated with treatment's side effects (35.4\%). Pain prevented patients fulfilling of their family obligations in $29.2 \%$ cases. Physical tiredness and discomfort was present in $83 \%$ of patients, which was attributed in $67 \%$ of them to the treatment received. Most patients considered they were below their best condition. Regarding family and social welfare, $10 \%$ felt that their partner was distancing from them. Considering emotional wellbeing, $20.4 \%$ reported sadness and $14.3 \%$, felt that their medical condition was not improving. In relation to functional well-being, $29.2 \%$ had enough or much ability to work compared to $54.5 \%$ of the subgroup with non-active CGvHD.

Conclusion: Development of cGvHD influences negatively most areas, with patients suffering emotional stress that leads them to feel sadness and hopelessness. And although they feel supported by their family and social environment. Finally, patients reported to have reduced ability to work and the feeling of not having an acceptable quality of life.

Disclosure of Interest: None declared.

\section{NP046}

Treatment of acute leukemia in pregnant patients and impact of stem cells transplantation on female fertility case reports

K. Kabatova Maxova ${ }^{1}$, M. Marxova ${ }^{1, *}$, P. Hlinkova ${ }^{1}$, A. Jungova ${ }^{1}$, E. Bystricka ${ }^{1}$, S. Vokurka ${ }^{1}$, P. Jindra ${ }^{1}$

${ }^{1}$ Haematology-oncology department, University Hospital Plzen, Plzen, Czech Republic

Introduction: There is only case-report based experience in respect to acute leukemia treatment in pregnant patients. Any such a case must be individually considered and decision on chemotherapy administration or delay must reflect consequences potentially imposed on the life and health of mother and the fetus/child. In the first trimester, therapeutic abortion is recommended because of high risk of spontaneous abortion or serious birth defects and malformations. In the third trimester, some chemotherapy protocols and drugs can relatively safely be administered to pregnant patients, however, decision can usually be made to induce premature birth, or to delay chemotherapy after the child birth, and corticosteroids may relatively safely be used in pregnant ALL patients. Besides these issues, intensive chemotherapy and/or stem cells transplantation (SCT) may impose significant risk of infertility on female survivors. In any case, cooperation with the gynecology-obstetrics, pediatricians (neonathology) and psychologist is crucial. There are several case reports reflecting this important medical and nursing problem in our transplant centre patients.

Method: Case report 1: 31-yaer old patient, diagnosed with AML in $12 / 2012$ while in the $11^{\text {th }}$ week of pregnancy, therapeutic abortion performed early in 12/2012 and intensive chemotherapy was administered, allogeneic SCT (Bu/CY/ATG) was performed in $3 / 2013$, he patient is healthy and alive. Case report 2: 41-year old patient, diagnosed with $A M L$ in 9/2012 while in the $11^{\text {th }}$ week of pregnancy, therapeutic abortion performed early in 9/2012 and intensive chemotherapy was administered, allogeneic SCT (FLU/Mel) performed in $12 / 2012$, patient is healthy and alive.

Results: Case report 3: 26-year old patient, diagnosed with ALL in $12 / 2006$ while in the $25^{\text {th }}$ week of pregnancy, premature birth of a healthy boy (C-section) performed in $12 / 2006$ and intensive chemotherapy was administered, the patient died in 1/2009 due to multiorgan failure and invasive pulmonary Aspergillosis after allogeneic SCT performed in 8/2008 because of ALL relapse. Case report 4: 39-year patient diagnosed with severe aplastic anemia in $3 / 2010$, treated with allogeneic SCT (cyclophosphamid/ATG) in 4/2010, pregnancy in $2 / 2014$, birth of healthy daughter in $10 / 2014$, the patient is healthy and alive. In our cohort of transplanted patients, we have 12 women with successful pregnancy and stem cells transplantation in their history.

Conclusion: Pregnancy and newly diagnosed acute leukemia or transplantation imposes high stress both on the patient and the health-care providers, because of large scale of consequences. Positive results, however, may also be observed.

Disclosure of Interest: None declared.

\section{NP047}

Sexuality in Hematopoieti Stem Cell Transplant Patients Slovenian Experience

P. Rihtarsic ${ }^{1, *}$, M. Sever ${ }^{1}$, M. Tinkara ${ }^{1}$, I. K. Skoda Gorican ${ }^{1}$

${ }^{1}$ Haematology Department, UMC Ljubljana, Ljubljana, Slovenia

Introduction: Sexuality in the hematopoietic stem cell transplant (HSCT) setting is infrequently discussed topic in everyday clinical practice. However, as a vital aspect of our wellbeing it is important not to avoid it. Sexuality as well as intimacy between the partners after HSCT should have the same attention as the disease and other health issues in the patient. Here we discuss consequences of treatment of hematologic diseases including HSCT on patients' sexuality with focus on information transfer from healthcare workers to the patients.

Method: We performed literature review and quantitative research method. The data was collected using an anonymous questionnaire at median 11.5 (range 6-20) months after the HSCT in 140 patients. The standard questionnaire used by Eeltink et al. focused on sexual activity, physical and psychological changes, fertility and quality of life.

Results: After the HSCT $64.4 \%$ patients reported changes affecting their sexuality. However, the information transfer on the topic from healthcare workers was inadequate and 55\% patients performed their own search for the required answers. Fifty five percent of them remained sexually active after the HSCT. Their partners were not included during sexuality survey 
in $82 \%$ which in some cases led to inadequate understanding of post HSCT relationship changes. Seventy seven percent of patients already had children so that fertility was not an issue. Also, in spite of reporting problems $92 \%$ of patients did not use any means to help them during their sexual intercourse. Overall, post HSCT changes in sexuality had consequences on patients' quality of life and were scaled on Likert's scale as 3 - average.

Conclusion: Patients after hematopoietic stem cell transplant need additional information on sexuality. Individually oriented health education for patients and their partners needs to be established. Additional research on larger patient population is required to elucidate further problems in this field. We need additional patient information in Slovenian language as current sources are lacking or inadequate.

Disclosure of Interest: None declared.

\section{NP048}

To review the differences in intravenous calcium prescribing, during peripheral blood stem cell collection, between Edinburgh and Glasgow Clinical Apheresis Units M. Anderson 1,*, J. Fotheringham ${ }^{1}$, S. Murdoch ${ }^{7}$, J. Sinclair? S. Taylor ${ }^{2}$

${ }^{1}$ Clinical Apheresis Unit. Royal Infirmary, Scottish National Blood Transfusion Service, Edinburgh, ${ }^{2}$ Clinical Apheresis Unit. Glasgow, Scottish National Blood Transfusion Service, Glasgow, United Kingdom

Introduction: As part of an on-going audit programme within the Scottish National Blood Transfusion Service (SNBTS), it was noted that the Edinburgh apheresis unit had more citrate related adverse events, than the Glasgow apheresis unit. Both had their own local practice for the prescribing of intravenous (I.V.) calcium gluconate, for peripheral stem cell collections, and so a proposal was made to see how each centre dealt with the problem of citrate sensitivity and whether that would explain the disparity between the two centres.

Method: A standardised data collection document was developed and the study was carried out over a six month period (April - September 2014). Due to the small numbers of paediatric patients this data was not collected.

Data collected:

- Number of procedures carried out within the six months

- Standard I.V. calcium gluconate prescription used in each unit

- Number of times calcium gluconate was prescribed

- Whether the calcium gluconate was prescribed for relief of symptoms or prophylactically

- AC infusion rates and ratios

- Inlet speed

Results: Over a six month period a total of 150 peripheral stem cell harvests were carried out (75 in Edinburgh and 75 in Glasgow) with I.V. calcium gluconate used by Edinburgh 28 times (37\%) and by Glasgow 5 times (6\%). This was further broken down in to symptomatic or prophylactic prescription. There were a few differences in practice between the Edinburgh and Glasgow units.

Firstly, Glasgow corrects lower calcium and magnesium levels, pre procedure, calcium gluconate would then be prescribed for the duration of the procedure, however this had not been required (for any patient) over this period.

Secondly, Glasgow run their anti-coagulant ratio and anticoagulant rate slightly different to the unit in Edinburgh, but this was not felt to be significant.

Lastly, it was discovered that there were differences in the administration of calcium gluconate.

Conclusion: Unfortunately the study failed to explain the variation in citrate related adverse events between the two units. Initially, when it was realised that the Glasgow unit corrected low calcium and magnesium levels pre procedure this seemed like the most likely reason. However Glasgow had not found this necessary over the period of the study. Another variance between the two units was in relation to the anti-coagulant rate and ratios used. Although these were not felt to be significant, Edinburgh may adopt the Glasgow parameters for a period of 8-12 weeks and observe the results. Alternatively the whole study could be repeated to verify/ compare with the previous results.

The study shows the variation between the stem cell collecting units. Therefore the SNBTS will now look at producing guidelines for prescribing calcium gluconate and at standardising machine parameters for collection procedures.

Disclosure of Interest: None declared.

\section{NP049}

Living the Vision: Developing and Implementing the Nurse Care Coordinator (NCC) Role in the BMT Department

N. Stein ${ }^{1, *}$, C. Shamir ${ }^{1}$, R. Zelker ${ }^{1}$, O. Shapir ${ }^{1}$, R. Radiano ${ }^{1}$, Y. Frank-Kamenetsky', M. Ben-Izhak ${ }^{7}$

${ }^{1}$ Bone Marrow Transplantation, Hadassah Medical Center, Jerusalem, Israel

Introduction: Given the dynamic environment of the Bone Marrow Transplantation (BMT) department, nursing professionals must continually assess treatment challenges and advocate for patients in a multidisciplinary team, while adapting to the complicated needs of each patient and family. In 2005-2006, with our eyes to the future, a working group of nursing leaders at Hadassah Medical Organization (HMO) redefined their vision of the nurse's role. Our department was chosen as a pilot department for the implementation process of the nursing vision.

Method: A hospital steering committee set 16 core organizational parameters to define the NCC model. Central among these parameters were: patient and family education, leadership in the multidisciplinary team, and management of intensive needs upon patient discharge. As part of the divisional initiative, working groups within our department were established to define the NCC role and activities. These working groups further adapted the core parameters to the BMT patients' needs. The BMT parameters included: symptom management, guidance in living with central line, identification of carriers of resistant strains of bacteria, education for oral hygiene, coordination of home IV treatment and more. These parameters also served as a tool for evaluation of nursing performance in the transition to NCC.

Results: The working groups in BMT developed specialized care plans for the BMT patients' needs, such as care plans for neutropenia, mucositis, special medication regimes, and more. The NCC in our department is a registered nurse with at least 6 months of clinical experience, and has overarching responsibility for comprehensive care, peer support, and ensuring adherence to standards of care. The NCC arranges visits of dietitians, physiotherapists, social workers, psychologists, and other health professionals. The NCC actively participates in staff meetings, planning meetings and daily and weekly physicians' rounds. The NCC training program is in accordance with the adapted parameters that were formed by the developing groups in the department.

Conclusion: The NCC model is well absorbed in the BMT department; $63 \%$ (20/32) of the staff are certified as NCC. The implementation of the model has reinforced leadership within the nursing team and brought enthusiasm and initiative to the nursing practice. The model enables the NCC to take an integral and active part in decision-making and treatment. The model has promoted quality of patient care, contributed to team-building, and helped foster a positive atmosphere among team members and between staff members and patients. Adaptation of the model in our department has enhanced our ability to provide comprehensive, cutting edge, quality nursing care.

Disclosure of Interest: None declared. 
NP050

The Experience of Creation of the Transfusion Plateform in Hematology Day Hospital

O. Samsonova ${ }^{1, *}$, C. Hospital Gustems ${ }^{1}$, M. Michallet ${ }^{1}$, F. Barraco ${ }^{1}$, G. Salles ${ }^{2}$ on behalf of $M, H$. Labussiere Wallet ${ }^{1}$ on behalf of Christiane Cazac Chaverot, Christine Brechard

${ }^{1}$ Lyon Sud Hospital, MBJ, 'Lyon Sud Hospital, Hematology, Lyon, France

Introduction: Transfusion support is part of essential care in Hematology, aiming to improve patients' quality of life. Transfusion activity represents a sizable part of our specialty due to its impact on the pronostic of hematologic malignancies. It helps patients to maintain a good performance status but also to safeguard their social life.

Within the Hematology Day Hospital in our Lyon Sud Hospital Center, $25 \%$ of the global activity is represented by transfusion acts in MDS or acute leukemia elderly patients.

Throughout the years 2012, 2013 and 2014, the considerable increase in the number of transfusions $(+16 \%$, then $+21 \%)$ has represented a real source of disorganization for the nursing team, a lack of landmaks and considerable waiting time for mostly elderly patients.

A reorganization of transfusion care was peremptory.

Method: That lead to the creation of a "Transfusion Platform" in November 2014, within the Hematology Day Hospital.

This reorganization began with the nomination of a transfusion nurse, with a dedicated phone number. Electronic agendas which were up until then shared were split and a transfusion-specific agenda was created.

Also, a geographical "transfusion sector" was identified within the Day Hospital, with dedicated beds.

This platform aims to:

- identify transfusion threshold for each patient and so transfusion indication according to pre-defined and consensual criteria

- re-define the making of appointments for transfusion support

- foster links with "Etablissement Français du Sang" (French Establishement of Blood) with anticipated blood and platelets orders, aiming to a quick delivery of blood products

- obtain a better follow-up and a better knowledge of polytransfused patients and of transfusion-related complications

Results: After 3 months of test, we wanted to know how the patients, who were accustomed to the old way, perceived this reorganization.

A satisfaction survey was finished, with written questionnaires. The answers led us to realize that $100 \%$ of patients thought it was a real plus to have a dedicated transfusion nurse. For them, this situation lead to "a better knowledge of patient record, better follow-up", "support", "protection", "reassurance", "better contact and relationship".

Conclusion: The creation of this platform allowed a real new dynamic around the transfusion act. In fact, we would like to invest ourselves in other projects, some of which are already in progress.

Thanks to the involvement of the team, and to the cooperation of nursing and medical professionals from other French Hematology Centers, we created a new national tool "Information book for the polytransfused patient", in printing. After reasoning about transfusion-related hemosiderosis, we are aiming to improve, and then uniform, the medical and nursing care for these side effects, with an iron chelation protocol, as to standardize medical practice.

Disclosure of Interest: None declared.
NP051

A Single Institution Experience in Applying for JACIE Accreditation: How to Cross the Barriers?

R. Kawas ${ }^{1,{ }^{*}}$, R. Nehmeh ${ }^{1}$, S. Okaily ${ }^{1}$, N. Takkoush ${ }^{1}$, A. Zahreddine ${ }^{1}$, A. Bazarbachi ${ }^{1}$

${ }^{1}$ AMERICAN UNIVERSITY OF BEIRUT MEDICAL CENTER, Beirut, Lebanon

Introduction: Joint Accreditation Committee ISCT \& EBMT (JACIE) is the most prestigious accreditation that ensures high quality of care for recipients and donors of Hematopoietic Stem Cell Transplantation (HSCT). It sets professional standards of practice that increase consistency in healthcare delivery which in turn improves patient care outcomes and enhances the satisfaction of both providers and HSCT recipients. Our dedicated HSCT multidisciplinary team at the American University of Beirut Medical Center has applied for this prestigious accreditation. However, this can be a challenging yet an exciting journey where multidisciplinary efforts should be in place to cross potential barriers.

Method: To identify barriers and areas for improvement in our HSCT program, a task team composed of dedicated healthcare professionals from various disciplines was formed. The team members met on a weekly basis to review JACIE standards and perform self-assessment to identify potential gaps. During the meetings, the multidisciplinary task team members placed extensive efforts to identify potential barriers and suggest strategies for improvement.

Results: After reviewing JACIE requirements and evaluating the program performance, the multidisciplinary team identified two major barriers. The absence of a quality management (QM) model that delineates the quality structure and processes in the program on one hand; on the other hand, the inadequacy of standards of practice guidelines that covers all the HSCT clinical procedures. To embed a QM model in our program, a quality manager has been hired to track and perform the quality initiatives based on JACIE requirements. The three major milestones that were achieved in timely manner were creating an audit plan for key procedures in the program, collecting and reporting quality indicators on a quarterly basis, and setting a benchmark reference for each indicator. Additionally, we started to conduct quarterly quality management meetings to discuss the program performance against the new standards and set action plans for any observed underperformance for each indicator. Moreover, the task team made an assignment among members to complete all the standards of practice guidelines based on both the latest evidence-based knowledge and JACIE requirements.

Conclusion: The new QM model was successfully implemented in the program that reflected positively on staff satisfaction and patient care outcomes. Additionally, the clinical practice guidelines were completed and made accessible to the entire multidisciplinary team. Crossing these barriers to achieve JACIE accreditation has offered a comprehensive excellence and consistency in healthcare delivery throughout the transplant processes. We recommend that other centers adopt similar strategies during their exciting journey towards JACIE accreditation.

Disclosure of Interest: None declared.

\section{NP052}

The Growth of a NHS Joint Ventures Private Haematology and Transplant Unit

S. cleaver ${ }^{1}$, R. Clout ${ }^{1, *}$, A. Newson ${ }^{1}$, J. Shearn ${ }^{1}$

${ }^{1}$ Christie Clinic Haematology and Transplant Unit, THE CHRISTIE, Manchester, United Kingdom

Introduction: The Christie Clinic is a joint venture between Hospital Cooperation of America (HCA) and The Christie NHS Foundation Trust. These two providers came together in 2010 to provide a cancer treatment service for private patients.This partnership provides additional revenue for the Trust, which ultimately benefits NHS patients with $£ 11.3$ million invested by 
The Christie Clinic into The Christie NHS Trust. The Haematology Transplant Unit (HTU) is the only dedicated private inpatient Haematology service outside of London. The HTU has 6 single rooms, of which 5 are HEPA filtered rooms specifically designed for Haematology and Transplant patients. Method: The Christie Hospital has recently undergone a JACIE re-inspection. The stem cell transplant programme encompasses the NHS transplant unit and now the private patient unit. This helps to enhance communication between both clinical areas, and the NHS collections and processing facilities that are utilised by the private ward. The business developed rapidly so has the need to increase staffing numbers. There is now a senior sister, 2 junior sisters and 8 staff nurses on HTU. There were early challenges of low activity and therefore maintaining skills and staff morale was difficult. Staff are competent in the infusion of stem cells, fully trained in the administration of antibiotics for patients with suspected sepsis and the complex management of transplant patient care. There is an in house transplant training programme which has been attended by all staff. They are encouraged to undertake regular departmental audit to help improve the service and patient experience.

Results: The Christie Clinic HTU has now successfully performed 39 transplants in 5 years and over 200 patients have been admitted to the unit. There are potential plans to expand the HTU due to an increase in patient numbers. A haematology clinical fellow was appointed to help manage the complexities of patients and the patient load. In addition to this, there has been a recent appointment of a clinical nurse specialist (CNS) in haematology. The unit also has a clinical practice facilitator who ensures that the training is the standardised across the transplant programme.

Conclusion: Over the past four years the unit has seen steady progress. This increase in activity has aided the development of skills and knowledge within the team. At our recent JACIE inspection the private unit was positively received with recognition of good interactions between the NHS and private unit. Our on-going aim is to continue to support staff with our increasing activity by ensuring skills and knowledge are enhanced, therefore empowering staff with the confidence and capability to continue to deliver excellent patient care. We strive to maintain the personable service and individualised care that we are able to achieve through the operation of a small unit in order to enhance patient experience and satisfaction.

Disclosure of Interest: None declared.

\section{NP053 \\ Staff Knowledge in Oral Mucositis in Haematology \& Transplantation \\ R. Clout ${ }^{1, *}$, A. Dunn ${ }^{1}$ \\ ${ }^{1}$ Palatine' Ward \& Private HTU, THE CHRISTIE, Manchester, United Kingdom}

Introduction: Oral mucositis (OM) is a painful and debilitating complication of cancer therapy that can adversely affect patients' treatment regimens and quality of life (Quinn 2013). $\mathrm{OM}$ care is mainly supportive the use of the WHO oral toxicity scale on high-risk patients on a daily basis is required. Recommendations are basic oral care and good clinical practice. A ward audit in 2013 identified gaps in staff knowledge and the importance of mouth care and recognising OM. Gaps were identified and the clinical practice facilitator (CPF) implemented standardised training. This session included background to OM, examination of mouth, treatments and supportive care, and case examples using patient photos taken by medical illustration during various grades of mucositis or other oral complications.

Method: In 2015 a re-audit of nurses working in the inpatient transplant settings and assessment of knowledge on oral mucositis. This was to establish if improvements have been made, and future recommendations for improvements in patient care and nurses education. 55 staff had access to a questionnaire. There were a total of 26 responses $-47 \%$. Staff were asked a series of questions, the results were compared to 2013.

Results: The ward standard care is saline and difflam, adding in sodium bicarbonate when excess debris occurs. $46 \%$ of staff answered saline and difflam. Sodium bicarbonate was not mentioned by any respondents. The WHO oral toxicity score is used across the Trust to assess oral mucositis. 96\% staff answered this question correctly. In 2013 only 44\% answered this correctly. The areas of the mouth that should be examined including a picture diagram $96 \%$ of staff answered this question correctly. In $201327 \%$ answered correctly. A further question should two illustrations of patients with mucostis. Picture one - grade $280 \%$, picture two - grade $477 \%$. Significant improvement since 2013, only one person stated the grading of the pictures correctly. Is there anything in relation to oral care/mucositis care and treatment that you think we could do better? In 2013 staff recognised they need further education, whereas in 2015 the main responses were better patient education. Others suggestions included patient information leaflets, stricter administration of mouthwashes, not taking for granted that patients self-caring with mouthwashes, a drive on the importance of oral hygiene to patient and staff, packs on admission.

Conclusion: There is a marked improvement in knowledge since 2013. The results positively reflect on regular training that had occurred. There are still gaps in the knowledge of the products which should be used. The majority of staff have attended a session on oral care and mucositis. Patient photographs that have been used for teaching have since been developed into a ward poster of the WHO oral mucositis recognition tool. Training will contiune to focus on the importance of good patient and staff education and care.

Disclosure of Interest: None declared.

\section{NP054}

The successful Implementation of a Dedicated Vascular Access service for the Haemato-Oncology department S. Thomas ${ }^{1, *}$, J. Gururaj ${ }^{1}$, B. Olav ${ }^{1}$

"Haematology Outpatient, IMPERIAL COLLEGE HEALTHCARE NHS TRUST, LONDON, United Kingdom

Introduction: Central venous catheters (CVCs) are now the fundamental part of treatment and supportive care of patients with cancer. The use of CVCs has greatly improved the quality of care in patients undergoing chemotherapy and stem cell transplants. In 2012 the Haematology unit at Hammersmith Hospital introduced a nurse led peripherally inserted central venous catheter (PICC) service, however the nurses did not have dedicated days or timeslots for PICC insertion. As the number of referrals increased the workload became more difficult to manage in addition to the Day care duties.

Method: In November 2014 it was therefore decided that two of these experienced PICC placers from haematology day unit should work one day a week each as a dedicated vascular access nurse. By doing this it enabled them to plan and utilise time more efficiently and also to accommodate more patients. The amount of patients having PICC lines inserted has gone up from 46 in 2013 to 137 in 2014 and from March 2015 to October 64, as a result of having a dedicated vascular access team.

Results: The availability of knowledgeable and experienced nurses within the speciality results in fewer complications, improved success rate, overall better patient satisfaction, with continuity of care and cost reduction. There has been a marked decrease in waiting time for PICC insertion, which prevents delays in starting chemotherapy and other treatments. By having dedicated days the rest of the multidisciplinary team can plan and prepare patients' treatments in advance.

Many studies have proven that nurses need to have skills and knowledge about the care of CVCs to ensure reduction in common complications like infections and occlusions. The vascular nurse educates junior nurses regarding CVC care and 
assesses their competencies and is readily available when trouble shooting is required. Development of a dedicated vascular access nurse role within haemato-oncology has helped to us maintain high standards in the care of CVCs.

Conclusion: We will therefore recommend that a Haematology vascular access practitioner is incorporated into the haematology team.

Disclosure of Interest: None declared.

\section{NP055}

The recognition and management of challenges surrounding Paediatric Apheresis

T. J. Segrott ${ }^{1, *}$, S. Schnellmann-Smith ${ }^{1}$

${ }^{1}$ Paediatrics, Royal Marsden Nhs Trust, Sutton, United Kingdom

Introduction: Many paediatric cancers can be cured with an autologous haematopoietic stem cell transplant in combination with other treatment modalities. However, there are many difficulties encountered with paediatric stem cell harvesting. Each patient presents new challenges which need to be addressed to successfully complete a stem cell harvest.

These complexities are exacerbated by the relatively small number of paediatric patients compared to adults.

Some of these challenges can be pre-empted and addressed proactively to allow the patient and family to encounter a positive apheresis experience.

Aim: To highlight the challenges experienced during paediatric apheresis procedures and implement strategies to minimize these.

Method: Between January 2014 and December 2015 our centre performed 26 paediatric stem cell collections. Data regarding age, diagnosis, mobilisation therapy, venous access, citrate toxicity and stem cell yield were collected and retrospectively analysed.

Gender

- Male 11

- Female 14

NB: One patient was harvested on two separate occassions.

The mean age was 7.16 years

Of these patients the diagnoses were:

- Neuroblastoma: 14

- Wilms: 4

- Hodgkins Lymphoma: 3

- Medullablastoma: 3

- Anaplastic Large Cell Lymphoma: 1

Results: Common challenges encountered were as follows:

- Difficulties in mobilising stem cells

- The dilemma of venous access

- Patient/Family education/reduction of anxiety

- Removal of venous access devices

- Working autonomously and maintaining competencies

Conclusion: There are numerous challenges associated with apheresis procedures in this patient population.

Poor mobilisation remains a problem; however, this has greatly improved since changing the administration of GCSF from intravenous to subcutaneous.

Having followed recommendations, our centre uses femoral high flow double lumen catheters which has minimised procedural interruptions. However, removal of these remains traumatic with limited alternative resolutions.

Families have expressed wanting more information prior to admission for the harvest, implementing patient/family education could improve patient preparation and reduce anxiety. Currently an age appropriate picture book is being produced to address this need. In addition involvement from play specialists may help to reduce anxiety and provide distraction for the patient.

Our centre provides a team of two paediatric apheresis nurses who work independently. However, due to the small patient population, exposure to the procedure is limited. Thus, meaning links with the adult service are paramount to maintain competency.

Disclosure of Interest: None declared.
NP056

The Role of the Anthony Nolan Nurse: The experience within 4 UK Transplant Centres'

C. M. Quillinan ${ }^{1, *}$, S. Paskar ${ }^{2}$, H. Leonard ${ }^{3}$, C. DeBiase ${ }^{4}$

${ }^{1}$ Haematology, The Chistie NHS Foundation Trust / Central Manchester Foundation Trust, Manchester, ${ }^{2}$ Haematology, Tyne Hospitals NHS Trust, Freeman Hospital, Newcastle, ${ }^{3}$ Haematology, The Royal Marsden NHS Foundation Trust, ${ }^{4}$ Patient Experience Team, Anthony Nolan, London, United Kingdom

Introduction: In September 2014, Anthony Nolan produced a policy report called A Roadmap for Recovery which examined the provision and availability of late effects services for posttransplant patients. As part of Anthony Nolan's commitment to the findings of this report they collaborated with NHS partners to fund and recruit three full time clinical nurse specialists (CNS) dedicated to the long term follow up and care of posttransplant patients. Patients receiving allogeneic haematopoietic stem cell transplants (HSCT) have notably higher rates of long-term side effects and late effects, with two thirds of adult long-term survivors having at least one chronic health condition and $20 \%$ having one severe or life threatening condition (Syrjala et al 2012). It has been reported that barriers to providing long term follow up include a lack of awareness of patient need, lack of education from both the patient and professional, lack of resources and inadequate communication between patient, transplant centre and community care (Majhail and Rizzo 2013).

Method: The method will look at the individual roles of three Anthony Nolan CNSs in 4 different UK transplant centres. Measurement of effectiveness will include both qualitative and quantitative data using a satisfaction questionnaire and a data collection dashboard, submitted monthly to Anthony Nolan. The three CNSs had staggered starts so the data is from September 2014 to September 2015 based on 26 active, clinical months between them.

\begin{tabular}{|c|c|c|c|c|c|}
\hline & $\begin{array}{l}\text { CNS1- } \\
\text { 13mths }\end{array}$ & $\begin{array}{l}\text { CNS2- } \\
\text { 9mths }\end{array}$ & $\begin{array}{l}\text { CNS3- } \\
\text { 4mths }\end{array}$ & TOTAL & $\begin{array}{c}\text { Ave per } \\
\text { mth }\end{array}$ \\
\hline $\begin{array}{l}\text { Number of } \\
\text { clinics }\end{array}$ & 192 & 90 & 49 & 331 & 12 clinics \\
\hline $\begin{array}{l}\text { Clinic time } \\
\text { (hours) }\end{array}$ & 595 & 330 & 201 & 1,126 & 43 hours \\
\hline New patients & 195 & 73 & 13 & 281 & $\begin{array}{l}11 \text { new } \\
\text { patients }\end{array}$ \\
\hline Follow-ups & 815 & 40 & 254 & 1,109 & $\begin{array}{l}43 \text { follow } \\
\text { ups }\end{array}$ \\
\hline $\begin{array}{l}\text { Non-clinic } \\
\text { family } \\
\text { contacts }\end{array}$ & 130 & 65 & 20 & 215 & 8 families \\
\hline $\begin{array}{l}\text { MDT } \\
\text { meetings }\end{array}$ & 75 & 80 & 2 & 157 & 6 MDTs \\
\hline
\end{tabular}

Results: CNS2 has also established a nurse-led DLI clinic and has facilitated 62 infusions in 9 months. In addition, she has implemented a satisfaction survey. As data collection is on-going, an updated abstract could be made available in the New Year.

Conclusion: The CNS role was created and funded by Anthony Nolan as a positive step to address the gaps across splintered and inconsistent care in post-transplant pathways. Over the next 5 years there will be an estimated 16,076 people living with the long-term effects of a stem cell transplant (BSBMT, 2015) and the introduction of the Anthony Nolan posttransplant CNS has demonstrated the value of developing local services whilst dramatically improving post-transplant care and education for patients and families. The collective goal is to provide a cohesive and consistent post-transplant 
pathway including efficacious and evidence based late effects services. The aim continues to be achieved; to improve the quality of life of every HSCT survivor.

Disclosure of Interest: None declared.

NP057

Low Well-Being and Higher Supportive Care Needs are Related to Increased Levels of Depressive, Anxiety and Physical Symptoms among Long-Term Survivors after Hematopoietic Stem Cell Transplantation. A Mixed Methods Analysis of the PROVIVO study

N. Gerwig ${ }^{1, *}$, M. Kirsch ${ }^{2}$, K. Fierz ${ }^{1}$, S. Beckmann ${ }^{1}$, S. De Geest ${ }^{1}$

${ }^{1}$ University of Basel, ${ }^{2}$ University Hospital Basel, Basel, Switzerland

Introduction: Long-term survivors after hematopoietic stem cell transplantation (SCT) experience serious late effects, which can compromise patients' well-being (WB) and increase their supportive care needs (SCN). However, no study examined patients' WB and SCN reported in clinical follow-ups and their association with clinical characteristics so far. Therefore the aims of this study were to describe SCT patients' long-term WB and $\mathrm{SCN}$, to examine the association of time after treatment, occurrence of cGvHD, psychological health and symptom occurrence with different domains of WB (general, physical, psychological, social, spiritual), and how these clinical parameters relate to $\mathrm{SCN}$.

Method: In this concurrent nested mixed methods analysis of the PROVIVO study data of $\mathbf{3 7 6}$ adult SCT long-term survivors from two Swiss University hospitals were analyzed with specific attention paid to patients' hand-written statements on their current WB and SCN during regular clinical follow-up. Deductive content analysis was used to explore a total of five forms of WB (general, physical, psychological, social, spiritual) and five forms of SCN (physical, psychological, social, spiritual and patients' questions). The qualitative data concerning SCN and WB was triangulated with selected quantitative clinical characteristics (time after treatment, occurrence of cGvHD, psychological health and symptom occurrence) by applying binary logistic regressions.

Results: The majority of patients had a positive general WB $(88 \%)$. However, complaints affecting physical WB (75\%) and psychological WB (52\%) were often reported. SCN were claimed with regard to physical (72\%), social (18\%) and psychological concerns (16\%). Reduced general WB was associated with more depressive (odds ratio (OR):287; confidence interval $(\mathrm{Cl}):(.121-.680) ; P=0.005)$ and physical symptoms (OR:.900; Cl: (.823-.984); $P=0.021)$. Negative physical WB was related to increased physical symptoms (OR:.880; Cl: (.815-.951); $P=0.001)$. Negative psychological WB was associated with more emotional/ cognitional symptoms (OR:.560; Cl: (.352-.889); $P=0.014)$. Reduced social WB was associated with increased depressive symptomatology (OR:.434; Cl (.231-.814); $P=0.009)$ while anxiety symptoms were related to more physical (OR:1.365; Cl: (1.125-1.656); $P=0.002)$, social (OR:1.199; Cl: $(1.016-1.416) ; P=0.032)$ and psychological SCN (OR:1.283; Cl: (1.062-1.548); $P=0.009)$.

Conclusion: Findings of this study implicate that patients with depressive, anxiety and physical symptoms are at particular risk of reduced WB and more SCN. These results provide knowledge necessary to implement patient-tailored follow-up care services to improve survivors' WB and fulfill their SCN.

Disclosure of Interest: None declared.

\section{NP058}

Post-discharge life adaptations of spouses of allotransplanted male patients

N. Yokota, ${ }^{1, *}$, T. Kamimura ${ }^{2}$, T. Miyamoto ${ }^{3}$, M. Oda $^{4}$

${ }^{1}$ Department of Nursing, ${ }^{2}$ Department of Hematology, Harasanshin Hospital, ${ }^{3}$ Department of Medicine and Biosystemic Science, Kyushu University Graduate School of Medical Sciences, ${ }^{4}$ Graduate School of Health and Welfare Sciences, International University of Health and Welfare, Fukuoka City, Japan

Introduction: The aim of this study was to characterize the process of adaptation experienced by the female spouses of male patients who underwent allogeneic hematopoietic stem cell transplantation (allo-HSCT) following discharge.

Method: We conducted semi-structured interviews with six spouses and questioned them about the difficulties experienced in daily life following the discharge of their husband, and their coping strategies. We descriptively analyzed the interview data using the modified grounded theory approach. Results: We extracted the category of 'habituation of combating communicable diseases' from subcategories "responsibility for making effort," "management of everyday life, " "confidence derived from experience" and "habituation in the family." We also extracted the other category "change in anxiety and concern' from "perception of differences in attitude in daily life between husband and wife," "change in the sense of values," "finding a point of compromise in each family," "benefit from allotransplant," and "persistent anxiety." 1. Habituation of combating communicable diseases

In the early discharge period, spouses experienced many various difficulties in daily life with understanding that they had responsibility for making effort in combating communicable disease. Spouses repeatedly managed everyday life with their husbands resulting in habituation of combating communicable disease in the family, while self-confidence was derived from these experiences (Figure 1). 2. Change in anxiety and concern

Spouses perceived differences in attitude in daily life between husband and wife with feeling irritated at her husband who lazed around all day, at the beginning of post-discharge life. However, spouses found a point of compromise in each family while they changed their own sense of values. Consequently, they became aware of benefit from allo-HSCT, resulting in post-discharge life adaptations. Nevertheless, spouses persistently felt anxiety about financial hardships and complications such as infections and GVHD even after long periods of time rom allo-HSCT (Figure 1).

Conclusion: The females habituated themselves to combating communicable diseases by gaining self-efficacy so that they reconciled a new, fixed lifestyle with their husbands following allo-HSCT. Although their anxiety and concerns changed over time, they had persistent anxiety about both their husbands' health, including the risk of relapse or GVHD, and financial hardships.

Disclosure of Interest: None declared.

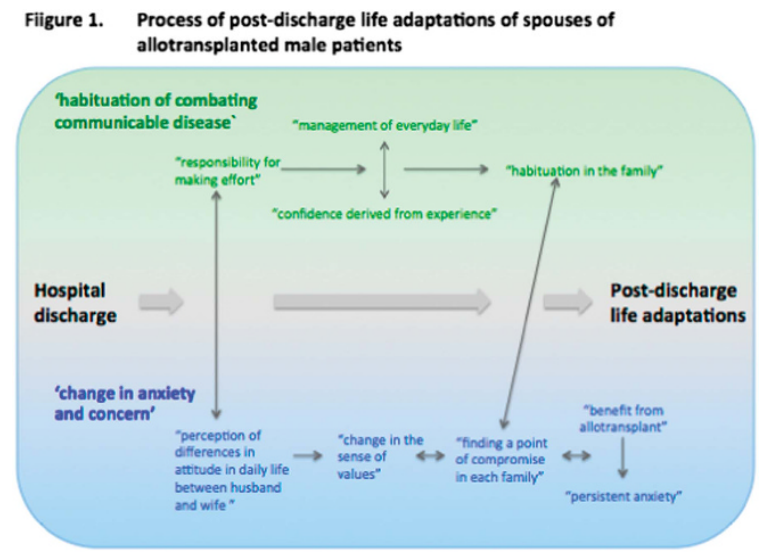


NP059

Knowledge of nurses and physicians about chemotherapy induced nausea and vomiting (CINV) in the setting of hematopoietic stem cell transplantation (HSCT): the Rome Transplant Network (RTN) experience

A. Tendas ${ }^{1, *}$, L. cupelli ${ }^{1}$, M. R. mauroni ${ }^{2}$, F. di piazza ${ }^{2}$, D. saltarelli ${ }^{3}$, I. carli ${ }^{3}$, A. chierichini ${ }^{3}$, C. melfa ${ }^{4}, M$. A. surano ${ }^{4}$, O. annibali ${ }^{4}$ F. sollazzo ${ }^{5}$, M. piedimonte ${ }^{5}$, E. conte ${ }^{5}$, F. marchesi ${ }^{6}$, C. viggiani ${ }^{6}$, A. pignatelli ${ }^{6}$, T. dentamaro ${ }^{7}$, A. P. perrotti ${ }^{7}$, P. de fabritiis ${ }^{\prime}$, W. arcese $^{2}$ on behalf of Rome Transplant Network Quality of Live Working Party

${ }^{1}$ Hematology, S: Eugenio Hospital, ${ }^{2}$ Hematology, "Tor Vergata" University Hospital, " Hematology, S. Giovanni Addolorata Hospital, ${ }^{4}$ Hematology, University Campus Bio-Medico, ${ }^{5}$ Hematology, S. Andrea Hospital, ${ }^{6}$ Hematology and Stem Cell Transplantation Unit, Regina Elena National Cancer Institute, rome, Italy

Introduction: Proper CINV management requires accurate knowledge and attitudes expertise. The purpose of this study was to investigate the knowledge and attitudes of Italian HSCT nurses and physicians concerning CINV management.

Method: The sample consisted of 96 nurses and physicians in the six HSCT centers of the RTN. A three parts anonymous questionnaire was designed for the purpose of this study; part 1: mechanisms, prevention and management of CINV (14 multiple choice questions); part 2: self-evaluation about professionals CINV knowledge (1 numerical-rating-scale question ranging 0-10); part 3: background information concerning professionals ( 5 questions: age, length of working in hematology, length of working in HSCT Unit, nurse or physician role, membership of the Quality of Life Working Group of the RTN). Survey was announced by e-mail to each professional and it was active from 3/7/2015 to 3/8/2015; remainders were sent weekly by e-mail; questionnaire was anonymously responded by accessing to an electronic module.

Results: Forty-one professionals completed the questionnaire (42\% response rate). Age of the responders was 40 (22-66) years; 31 nurses, 8 physicians, 1 undisclosed. The average correct response rate for each responder was 51.8 (range 14.3 - 92.9) \%; the average correct response rate for each question of part 3 was 52.2 (range 12.5-97.5) \%. Further analysis of knowledge scores by responders background characteristics showed that the CINV knowledge was positively correlated to length of working in Hematology units, physician role and membership of the Quality of Life Working Group (Table 1). Correlation between self-evaluation of CINV knowledge and knowledge scores was good: average correct response rate of responders self-evaluating knowledge as poor (scale score $<6$ ) and good (scale score $>=6$ ) was $44.1 \%$ and $60.3 \%$, respectively $(P<0.01)$.

Table 1. Correlation between responders background characteristics and CINV knowledge

\begin{tabular}{lccc}
\hline & \multicolumn{3}{c}{$\begin{array}{c}\text { average correct } \\
\text { response rate }\end{array}$} \\
\hline Length of working & $>8$ years & $59.8 \%$ & $<0.01$ \\
in Hematology & & & \\
& $<=8$ years & $44.3 \%$ & \\
Role & Physician & $70.5 \%$ & $<0.01$ \\
RTN QoL WP & Nurse & $46.8 \%$ & \\
membership & Yes & $64.4 \%$ & $<0.01$ \\
& No & $46.4 \%$ & \\
\hline
\end{tabular}

Conclusion: The findings suggest that area of improvement of knowledge on CINV among HSCT staff exists; training programs for HSCT staff should address CINV issue, in order to improve level of knowledge about this highly discomforting symptom.

Disclosure of Interest: None declared.

NP060

Nurses' guidelines for the management of Hepatic

Veno-Occlusive Disease following Hematopoietic Stem Cell Transplant: the development of monitoring flow-chart

L. Orlando ${ }^{1, *}$ on behalf of GITMO, S. Botti ${ }^{2}$, V. De Cecco ${ }^{3}$, G. Gargiulo ${ }^{4}$

${ }^{1}$ Division of Clinical Haemato-Oncology, EUROPEAN INSTITUTE OF ONCOLOGY, Milano, ${ }^{2}$ Division of Clinical Haemato-Oncology, ASMN-IRCSS, Reggio Emilia, ${ }^{3}$ Division of Pedriatric Clinical Haemato-Oncology, POLICLINICO SAN MATTEO, Pavia, ${ }^{4}$ Division of Clinical Haemato-Oncology, AOU FEDERICO II, Napoli, Italy

Introduction: Veno Occlusive Disease (VOD) or sinusoidal obstruction syndrome (SOS) is a life threatening complication in patients treated with hematopoietic stem cell transplantation. It is important to early recognize symptoms and signs of VOD which usually develop during the first 35-40 days posttransplant but may also develop at a later time. The diagnosis and the severity of VOD are based on the modified Seattle or the Baltimore clinical criteria. Nurses are involved at all management levels of VOD from the recognition of suggestive symptoms and signs during the monitoring phase such as weight gains, jaundice, nutritional and appetite changes as well as changes in the circumference of the abdomen. Therefore, they have to be efficient in the follow up of fluid and electrolyte balance, identify hemorrhagic manifestations and pain severity levels. The aim of this work is to establish risk oriented monitoring instrument that is specific, efficient, standardized and dynamic.

Method: Three main steps were followed to develop the monitoring chart:1) a working group of experts (EG) was established, 2) EG performed critical literature and SOP's review, 3) analysis and discussion of the results of a survey was conducted in GITMO network. EG identified the main criteria and factors essential for the development of the monitoring plan such as i) parameters and variables to monitor, ii) the timing and phases iii) frequency, and others. This was further refined and finalized in a monitoring flow chart based on a risk oriented approach for VOD assessment in patients during their transplant program.

Results: EG has defined three main phases for the assessment and the monitoring of patients during their transplant program. 1) Preconditioning phase of assessment: risk assessment for VOD should be generally estimated in a multidisciplinary approach. Patients should be risk categorized upfront accordingly; 2) Conditioning phase: the panel has put in place two monitoring levels according to risk assessment; 3) post-conditioning phase: nurses in this follow standard monitoring with careful evaluation of signs and symptoms according to the modified criteria of Seattle or of Baltimore. If no abnormalities occurs, standard monitoring continues as planned. In case of observing any positive criteria suggestive of VOD, physicians are alerted. Once the diagnosis is confirmed or highly suspected, the monitoring level is intensified.

Conclusion: Main objective of this working was to establish real life monitoring instrument that is based on data from the literature and also from the current clinical practice. The developed chart is dynamic following the evolution of patients' clinical condition. Expert panel has acknowledged the developed chart as the preferred instrument for nurses to use in the monitoring and management of VOD.

Disclosure of Interest: None declared. 
NP061

Management of Treatment-induced Diarrhoea associated with Bile Acid Malabsorption, in Multiple Myeloma patients

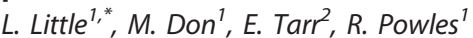

${ }^{1}$ Haemato-Oncology, ${ }^{2}$ Haemato-Oncology Pharmacy, Cancer

Centre London, Parkside Hospital, London, United Kingdom

Introduction: Diarrhoea is a common gastro-intestinal (Gl) toxicity associated with novel treatments for Multiple Myeloma (MM) including Lenalidomide and Bortezomib; routinely given during induction, relapse and maintenance phases. Any side effects, therefore, will have considerable Quality of Life (Q o L) implications for affected patients. Recent studies involving Lenalidomide ${ }^{1}$ have shown that this adverse effect (AE) may relate to Bile Acid Malabsorption (BAM) and can respond to treatment.

Rationale: Important treatments may be modified or discontinued due to diarrhoea thereby affecting outcomes, so early diagnosis and optimal treatment are essential. Bile Acid is produced in the liver during fat digestion, before being reabsorbed and recycled. In BAM this reabsorption in affected causing fluids to accumulate in the bowel, resulting in loose stools and diarrhoea. It is not yet understood why these treatments can cause BAM.

Method: Relevant patients are assessed to establish the cause of their diarrhoea, through clinical history and review. If found to be treatment-related, the severity is graded with reference to National Cancer Institute (NCI) Toxicity Criteria, and patients are tested for BAM. Diagnosis is by a simple non-evasive SeHCAT (75selenium homocholic acid taurine) scan to study the entro-hepatic circulation of bile acids; a capsule is administered orally, patients are scanned after 3 hours and then again after 7 days; a calculation of the 7 day retention is calculated. Normal retention is $>15 \%$. Treatment is with fat reduction diet alone (in mild cases), or with a bile acid sequestrant such as Coleveselam. Where indicated, with advice from dietician and/or the Gl team.

Results:

Patient Characteristics

Patient A. female, dob 31/01/1945. Diagnosed IgA Lambda MM 15.5.2008.

Number of prior treatment (Rx) lines: 7, including Autologous Stem Cell Transplants (ASCT): 2.

Patient B. male, dob 23/08/1944, Diagnosed Kappa Bence-Jones MM 3.3.2012.

Number of prior Rx lines: 2, including ASCTs: 1 (Tandem). Outcomes

Patient A

Patient B

MM Treatment

Baseline bowel

function

Treatment-induced

diarrhoea toxicity

score

SeCHAT Scan result

Treatment for BAM

Outcome

Patient $A$

Lenalidomide

Bortezomib
Normal bowel
function (previous
diarrhoea

Lenalidomide

Normal bowel

function

associated with

Lenalidomide)

$$
\text { 2-3 }
$$

Severe BAM: 0\% at day 7

Colesevelam hydrochloride $3.75 \mathrm{~g}$ daily Sustained reversal of diarrhoea symptoms, with immedicate effect.
Markedly abnormal $<1 \%$ at day 7 Colesevelam hydrochloride $3.75 \mathrm{~g}$ daily Sustained reversal of diarrhoea symptoms, within days.
Conclusion: As previously described, BAM is shown in patients receiving Lenalidomide; we also identify occurrence during treatment with Bortezomib. We can therefore recommend that all patients with Treatment-induced Diarrhoea while on these regimens should be tested for BAM.

Disclosure of Interest: None declared.

\section{NP062}

Acute Skin GvHD: nursing schedule evaluation tool for children

M. Canesi $i^{1, *}$, F. Pellegatta ${ }^{1}$, M. Della Ducata ${ }^{1}$ on behalf of Nursing staff of Pediatric Hematology - BMT Unit, Fondazione MBBM, Monza leaded by Ms Nadia Mandelli

${ }^{1}$ Pediatric Hematology - BMT, Fondazione MBBM - via Pergolesi 33 - Monza, Monza, Italy

Introduction: Graft versus Host Disease (GvHD) remains one of the main complications of haematopoietic stem cells transplantation. The incidence of acute GvHD (aGvHD) is 10-80\%. The skin is the organ most commonly involved on the onset of an aGvHD.There is no evidence abut nursing schedule to evaluate and monitor skin GvHD. There is no evidence avout the best nursing management of skin, according to GvHD stage. Method: Population: paediatric patients $(0-18 \mathrm{ys})$ undergoing haematopoietic stem cell transplantation. Limits: patients undergoing autologous transplant were excluded. No chronic skin GvHD has been considered. Method: licterature review; schedule creation; pilot study --> schedule has been tested in the BMT unit proposing (2 months); adaptation of the schedue according to results of pilot study. Each schedule was accompained by an anamnestic form about patients, both for disease and anagraphic datas.

Results: Schedule evaluation tool has to be filled at the first manifestation of aGvHD and every time there is something different in staging or treatment/management. The form collects datas about:

-aGvHD stage, considering body surface throung the Rule of $9 \mathrm{~s}$ (Paediatric adapted) and Lund-Browder diagram.

-aGvHD manifestation: rash, papule or others.

- where the aGvHD is (also graphically, using human bodies images, with details on hands and feet).

-if there are pain or itch (both of them evaluated with tools adapted for ages).

-if there are wounds aGvHD linked: dimensions and wound stage (necrosis, granulation, riepithelization)

-nursing management:

Conclusion: The schedule was useful and appreciated among nurses who referred about its clarity, immediacy and easy use. There were not anything similar to register nursing evalutation of skin aGVHD, so nurses think that it would be a useful and not redundant form. It was used even for comparison and uniformation between doctors' and nurses' evaluation of patients' skin in different day times.

It highlights incidence of skin aGvHD, gives a omogenous way of evaluate to all nursing staff and it registers which kind of treatment or management is performed, according to different GvHD stages and different patient's conditions.

In a second time, it could be shared among paediatric HSCT unit to collect datas about management of paediatric skin aGvHD and identify the best one, performing the best nursing care for patients and their families.

Disclosure of Interest: None declared.

\section{NP063}

Introducing intravenous (IV) Morphine PCA

(Patient-Controlled Analgesia) for in-patients dealing with mucositis issues

M. E. Ghannoo ${ }^{1, *}$

${ }^{1}$ Dept. of Internal Medicine (BMT ward), UNIVERSITY HOSPITALS GENEVA, Geneva, Switzerland

Introduction: Mucositis refers to a painful inflammatory and/or ulcerative lesions of the mucous membranes lining 
the digestive gastrointestinal tract. It is a common and often debilitating complication of post allogenic BMT (Blood and Bone marrow transplantation). Most in-patients suffer from mucositis, which is one of the common adverse effects (over $80 \%$ ) of post Allogenic BMT due to the conditioning programme. A PCA (Patient-controlled analgesia) is an electronic controlled device of self-administering pain relief when the patient presses a button. He is therefore protected from overdose as the PCA is programmed to deliver a dose of opioid at set intervals. If the patient presses the button sooner than the prescribed intake, the PCA will not operate as there is a set locked time. The aim of this study was to find the most appropriate mode of pain relief administration for these patients. The previous mode of pain-relief administration in the ward was by a syringe pump with a continuous rate of intravenous opioid medication.

Method: Multidisciplinary team building which involved medical and nurses staff of the BMT ward, staff of the post operative acute pain management team, and a specialized pharmacist in oncology care.

- Literature review on pain management using a PCA infusion for mucositis.

- Use of a PCA observation chart to monitor the known adverse effects of intravenous use of opioids (every 4 to 6 hours).

- Training of the general nurses by the post operative acute pain management team.

- Assessment and evaluation of the study every 3 months since the beginning of the PCA study by the multidisciplinary team.

Results: The test phase of the morphine PCA has been introduced to 14 patients from november 2014 to june 2015. The relevant indicators are: the number of days of morphine consumed by the patients (average:9), the amount of opioid dose requested and delivered over 24 hours combined to the total daily amount (median:29 mg, [0$153 \mathrm{mg}]$ ), the number of patients who had a continuous flow of opioid (8 patients), VAS (Visual Analog Scale) described by the patients, measurement of blood oxygen saturation, the respiratory frequency, sedation score measurement, nausea, pruritus and constipation observation. No patient experienced severe side effects. VAS however remained high but most patients have claimed their satisfaction in the pain management with a PCA.

Conclusion: The PCA seems to be an effective means of pain management for mucositis Nonetheless, the VAS assertion does not appear to be an accurate tool for the pain management under PCA. Other indicators have to be considered such as the information recorded on the PCA observation's chart. Medical prescriptions in the programming of the PCA have to be enhanced among the doctors. The introduction of the PCA required a strong leadership and guidance from the analgesia nurse referees and the head nurse of the BMT ward.

Disclosure of Interest: None declared.

\section{NP064}

Service Evaluation: To identify percentage weight loss in patients undergoing Stem Cell Transplantation (autologous and allogeneic)

N. Jones ${ }^{1, *}$

${ }^{7}$ Nutrition and Dietitian, ADDENBROOKES HOSPITAL, Cambridge, United Kingdom

Introduction: The nutritional management of patients undergoing stem cell transplantation can be challenging due to the side effects. This leads to them being at a high risk of malnutrition. It has been documented that a weight loss of $>10 \%$ is associated with worse clinical outcome. This review aimed to look at the percentage weight loss in patients undergoing autologous and allogeneic transplantation during 2012-2015. We currently place prophylactic naso-jejunal tubes in patients receiving cyclophosphamide/Total Body Irradiation, Buslfan/Cyclophosphamide, this review aimed to identify other chemotherapy conditioning regimens that would benefit from this approach.

Method: A dietetic database of all stem cell transplant patients seen by the dietitian since 2012, was used to gather weight information and conditioning regimens.

Results: Table One: Total Inclusion Figures

\begin{tabular}{lccccc}
\hline & 2012 & 2013 & 2014 & $\begin{array}{c}\text { 2015 (Jan to } \\
\text { mid August) }\end{array}$ & Totals \\
\hline autologous & 26 & 37 & 57 & 23 & 143 \\
allogeniec & 30 & 26 & 33 & 17 & 106 \\
myeloablative & 12 & 4 & 17 & 11 & 44 \\
& 68 & & 9 RIP during transplants & 293 \\
Exclusion & patients & & & \\
& & & 57 incomplete weights \\
\hline
\end{tabular}

Conclusion: This has highlighted that patients undergoing $\mathrm{Cy} / \mathrm{TBI}$ and $\mathrm{Cy} / \mathrm{Bu}$ conditioning transplants that have $\mathrm{NJ}$ tubes placed to enable enteral feeding through transplant have less weight loss than FLAMSA-Bu and Flu/TBI conditioning (see fig three below). This highlights the need for initiating prophylactic NJ feeding in this patient group. A multicentre study looking into feeding routes during stem cell transplant and the impact this has on weight loss would be recommended.

Disclosure of Interest: None declared.

\section{NP065}

Psychological intervention to alleviate distress in haematopoietic stem cell transplantation: A Phase $\mathbf{2}$ study M. Baliousis ${ }^{1}$, M. Rennoldson ${ }^{2}$, J. Mills ${ }^{3}$, L. Watson ${ }^{3,}{ }^{*}$, D. Dawson ${ }^{4}$, R. das Nair ${ }^{7}$

${ }^{1}$ University of Nottingham, ${ }^{2}$ Nottingham Trent University, ${ }^{3}$ Nottingham University Hospitals NHS Trust, Nottingham, ${ }^{4}$ University of Lincoln, Lincoln, United Kingdom

Introduction: Haematopoietic stem cell transplantation (HSCT) is an intensive procedure associated with considerable psychological distress particularly during the acute phase (first few weeks). This may also undermine physical well-being highlighting a need for psychological intervention. In light of limitations to the literature, such an intervention was developed drawing on psychological theory emphasising the role of patients' perceptions and coping strategies in maintaining distress during illness. The present study was a Phase 2 trial aiming to evaluate the feasibility of the design and the intervention whilst establishing the validity of the underlying theory in HSCT.

Method: Participants were adults recruited from consecutive referrals for HSCT at two transplant centres in the UK. Following consent, half were randomised to the intervention and half to treatment as usual. The intervention consisted of a 90- minute group held a few weeks prior to HSCT aiming at reducing negative perceptions of the procedure and fostering helpful coping using a combination of psychoeducation and Socratic enquiry. Psychological distress, HSCT perceptions, and coping were assessed at baseline (following consent), on the day of transplantation, two weeks, and then four weeks after transplantation.

Results: Of 99 eligible patients, 45 consented to participate with barriers primarily including competing priorities (other appointments), being unwell, travel distance, and insufficient time for obtaining consent prior to transplantation. Of those randomised to intervention, three patients with other unable to do so primarily due to insufficient time prior to transplantation, slow accrual for a group due to randomisation, and competing priorities. The probability of nonresponse increased from $11 \%$ on transplant day to $20 \%$ at the final 
follow up and was associated with higher stress earlier on. Anxiety peaked two weeks following transplantation but decreased thereafter whilst depression continued to increase throughout the study. Perceiving HSCT as having negative consequences, with prolonged timelines, higher symptom severity, cause for concern, and emotional impact together with limited understanding predicted higher distress throughout the period. Use of unhelpful coping styles (disengagement, denial, etc.) but also planning, emotional, and instrumental support also predicted higher distress.
Conclusion: The findings illustrated complex psychological needs during the acute phase of HSCT and the relevance of HSCT perceptions and coping in the manifestation of distress. However, they also revealed considerable barriers to employing a randomised trial during this period and to intervention uptake. These highlighted the need to explore alternative methods of evaluation, close integration of psychological intervention with routine care, and psychological input throughout the period.

Disclosure of Interest: None declared. 